\title{
Synthetic Strategy for Preserving Sky-Blue Electrophosphorescence in Square-Planar Pt(II) Complexes
}

\author{
Yu Kyung Moon, ${ }^{a \dagger}$ Jin-Suk Huh, ${ }^{b \dagger}$ Sonam Kim, ${ }^{a}$ Sinheui Kim, ${ }^{a}$ Seung Yeon Yi, ${ }^{a}$ Jang-Joo Kim, ${ }^{b *}$ \\ and Youngmin You ${ }^{a *}$
}

${ }^{a}$ Division of Chemical Engineering and Materials Science, Ewha Womans University, Seoul 03760, the Republic of Korea. ${ }^{b}$ Department of Materials Science and Engineering, Research Institute of Advanced Materials (RIAM), Seoul National University, Seoul 08826, the Republic of Korea.

${ }^{\dagger}$ These authors contributed equally to this work.

*To whom correspondence should be addressed: jjkim@snu.ac.kr (J.-J.K.); odds2@ewha.ac.kr (Y.Y.) 
Figure S1. $\quad$ UV-vis absorption spectra of toluene solutions containing $10 \mu \mathrm{M}$ free ligand $\quad$ S4

Figure S2. $\quad$ Cyclic and differential pulse voltammograms of PtSN1, PtSN2 and PtSN3 $\quad$ S5

Figure S3. $\quad$ Vibronic progressions of the photoluminescence spectra of (a) PtSN1, (b) PtSN2 and (c) PtSN3 obtained in Ar-saturated toluene

Figure S4. $\quad$ UV-vis absorption spectra of (a) PtSN1 and (b) PtSN3 recorded with increasing the concentration $(10 \mu \mathrm{M}$ to $1.0 \mathrm{mM})$

Figure S5. Photoluminescence excitation spectra of the monomer and excimer emissions of (a) PtSN1 and (b) PtSN2

Figure S6. Comparisons of the photoluminescence spectra of mCBP:TSPO1 (1:1, wt/wt) films doped with $8 \mathrm{wt} \% \mathrm{PtSN}$ dopants obtained at $100 \mathrm{~K}$ and room temperature

Figure S7. Photoluminescence decay traces of the monomer emissions of PtSN1 and PtSN3

Figure S8. Simulated external quantum efficiencies of a device with a configuration of ITO $(70 \mathrm{~nm}) / \mathrm{mCBP}: 6 \mathrm{wt} \% \mathrm{Re}_{2} \mathrm{O}_{7}$ (y nm)/mCBP $(10 \mathrm{~nm}) / \mathrm{mCBP}: T S P O 1: 8$ wt \% dopant $(20 \mathrm{~nm}) / \mathrm{TSPO} 1(\mathrm{x} \mathrm{nm}) / \mathrm{Mg}: \operatorname{Liq}(2 \mathrm{~nm}) / \mathrm{Al}(100 \mathrm{~nm})$

Figure S9. Deconvolution of the electroluminescence spectra of the devices of (a) PtSN1 and (b) PtSN2 recorded at $100 \mathrm{~cd} \mathrm{~m}^{-2}$

Figure S10. Angle-dependent photoluminescence intensities of 솓 $p$-polarized lights from mCBP:TSPO1 films doped with 2, 4 and 8 wt \% PtSN dopants

Figure S11. Photoluminescence spectra of mCBP:TSPO1 (1:1, wt/wt) thin films doped with $8 \mathrm{wt} \% \mathrm{PtSN}$ dopants recorded during continuous photoillumination under a wavelength of $325 \mathrm{~nm}$ from a $5 \mathrm{~mW}$ He:Cd laser

Figure S12. (a) Relative luminance $\left(L / L_{0}\right)$ and (d) driving voltage as a function of operation time of the PtSN devices driven at a constant current density of an initial value of $1 \mathrm{~mA} \mathrm{~cm}^{-2}$

Figure S13. $\quad{ }^{1} \mathrm{H}$ NMR spectrum $\left(300 \mathrm{MHz}, \mathrm{CD}_{2} \mathrm{Cl}_{2}\right)$ of di(3-iodophenyl)methane

Figure S14. $\quad{ }^{13} \mathrm{C}\left\{{ }^{1} \mathrm{H}\right\}$ NMR spectrum $\left(126 \mathrm{MHz}, \mathrm{CD}_{2} \mathrm{Cl}_{2}\right)$ of di(3-iodophenyl)methane

Figure S15.

${ }^{1} \mathrm{H}$ NMR spectrum $\left(300 \mathrm{MHz}, \mathrm{CD}_{2} \mathrm{Cl}_{2}\right.$ ) of $\mathrm{SN}_{1} \mathrm{H}_{2}$

Figure S16.

${ }^{13} \mathrm{C}\left\{{ }^{1} \mathrm{H}\right\}$ NMR spectrum $\left(126 \mathrm{MHz}, \mathrm{CD}_{2} \mathrm{Cl}_{2}\right)$ of $\mathrm{SN}_{1} \mathrm{H}_{2}$

Figure S17.

${ }^{1} \mathrm{H}$ NMR spectrum $\left(300 \mathrm{MHz}, \mathrm{CD}_{2} \mathrm{Cl}_{2}\right)$ of $\mathrm{SN} 2 \mathrm{H}_{2}$

Figure S18.

${ }^{13} \mathrm{C}\left\{{ }^{1} \mathrm{H}\right\}$ NMR spectrum $\left(126 \mathrm{MHz}\right.$, DMSO- $\left.d_{6}\right)$ of $\mathrm{SN}_{2} \mathrm{H}_{2}$

Figure S19.

${ }^{1} \mathrm{H}$ NMR spectrum $\left(300 \mathrm{MHz}, \mathrm{CD}_{2} \mathrm{Cl}_{2}\right.$ ) of $\mathrm{SN} 3 \mathrm{H}_{2}$

Figure S20.

${ }^{13} \mathrm{C}\left\{{ }^{1} \mathrm{H}\right\}$ NMR spectrum $\left(126 \mathrm{MHz}, \mathrm{CD}_{2} \mathrm{Cl}_{2}\right)$ of $\mathrm{SN} 3 \mathrm{H}_{2}$ 
$\begin{array}{llr}\text { Figure S21. } & { }^{1} \mathrm{H} \text { NMR spectrum }\left(300 \mathrm{MHz}, \mathrm{CD}_{2} \mathrm{Cl}_{2}\right) \text { of PtSN1 } & \text { S24 }\end{array}$

Figure S22. $\quad{ }^{13} \mathrm{C}\left\{{ }^{1} \mathrm{H}\right\}$ NMR spectrum $\left(126 \mathrm{MHz}, \mathrm{CD}_{2} \mathrm{Cl}_{2}\right)$ of PtSN1

Figure S23. $\quad{ }^{1} \mathrm{H}$ NMR spectrum $\left(300 \mathrm{MHz}, \mathrm{CD}_{2} \mathrm{Cl}_{2}\right)$ of PtSN2

Figure S24. $\quad{ }^{13} \mathrm{C}\left\{{ }^{1} \mathrm{H}\right\}$ NMR spectrum $\left(126 \mathrm{MHz}, \mathrm{DMSO}-d_{6}\right)$ of PtSN2

$\begin{array}{llr}\text { Figure S25. } & { }^{1} \mathrm{H} \text { NMR spectrum }\left(300 \mathrm{MHz}, \mathrm{CD}_{2} \mathrm{Cl}_{2}\right) \text { of } \mathrm{PtSN} 3 & \mathrm{~S} 28\end{array}$

Figure S26. $\quad{ }^{13} \mathrm{C}\left\{{ }^{1} \mathrm{H}\right\}$ NMR spectrum $\left(126 \mathrm{MHz}, \mathrm{CD}_{2} \mathrm{Cl}_{2}\right)$ of PtSN3 $\quad$ S29

Figure S27. Isosurface plots of the highest occupied molecular orbital (HOMO) and the lowest unoccupied molecular orbital (LUMO) of PtSN1 $\quad$ S30

Figure S28. Isosurface plots of the highest occupied molecular orbital (HOMO) and the lowest unoccupied molecular orbital (LUMO) of PtSN2

Figure S29. Isosurface plots of the highest occupied molecular orbital (HOMO), HOMO-1, and the lowest unoccupied molecular orbital (LUMO) of PtSN1

Table S1. $\quad$ TD-CAM-B3LYP Calculation Results for PtSN1 S30

Table S2. $\quad$ Report of the Cartesian Coordinates of the Optimized Geometry of PtSN1 S31

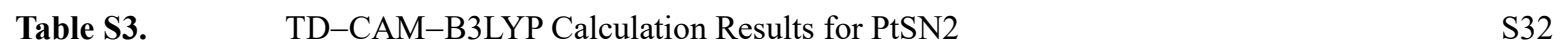

Table S4. $\quad$ Report of the Cartesian Coordinates of the Optimized Geometry of PtSN2 S33

$\begin{array}{lll}\text { Table S5. } & \text { TD-CAM-B3LYP Calculation Results for PtSN3 S35 }\end{array}$

Table S6. $\quad$ Report of the Cartesian Coordinates of the Optimized Geometry of PtSN3 S36

Table S7. Summary of the Photophysical Properties of the mCBP:TSPO1 Films Doped with the PtSN Compounds $\quad$ S38 
(a)

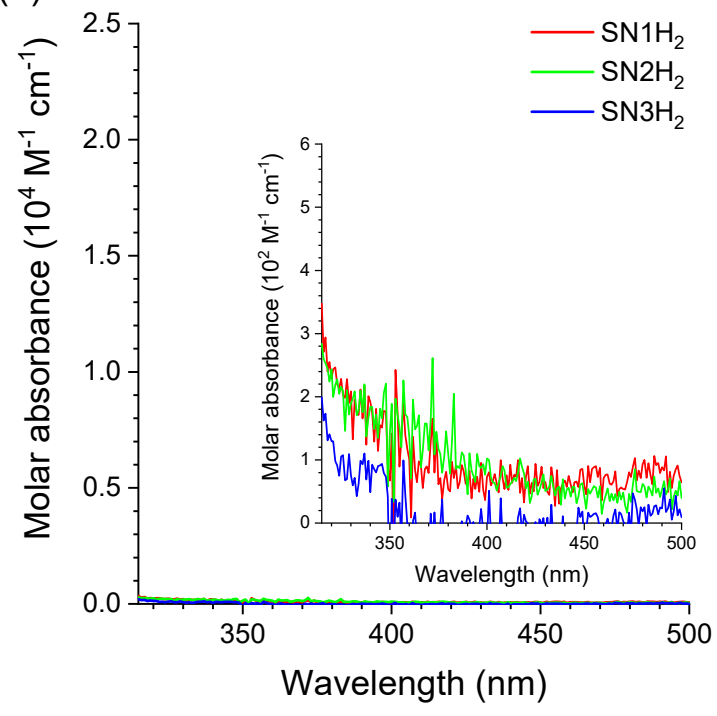

(b)

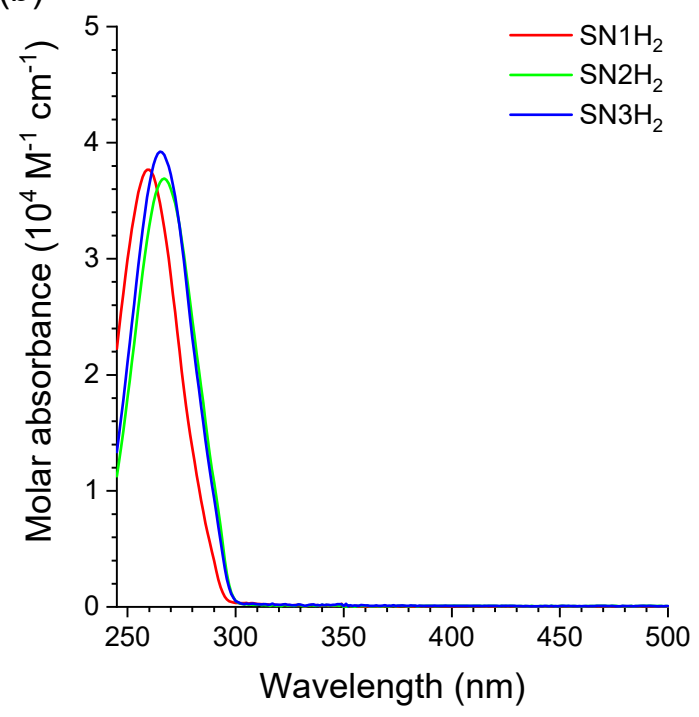

Figure S1. UV-vis absorption spectra of the toluene (a) and $\mathrm{CH}_{2} \mathrm{Cl}_{2}$ (b) solutions containing $10 \mu \mathrm{M}$ free ligand $\left(\mathrm{SN} 1 \mathrm{H}_{2}, \mathrm{SN}_{2} \mathrm{H}_{2}\right.$ and $\left.\mathrm{SN}_{3} \mathrm{H}_{2}\right)$. The inset graph in (a) is a magnification of the spectra. 


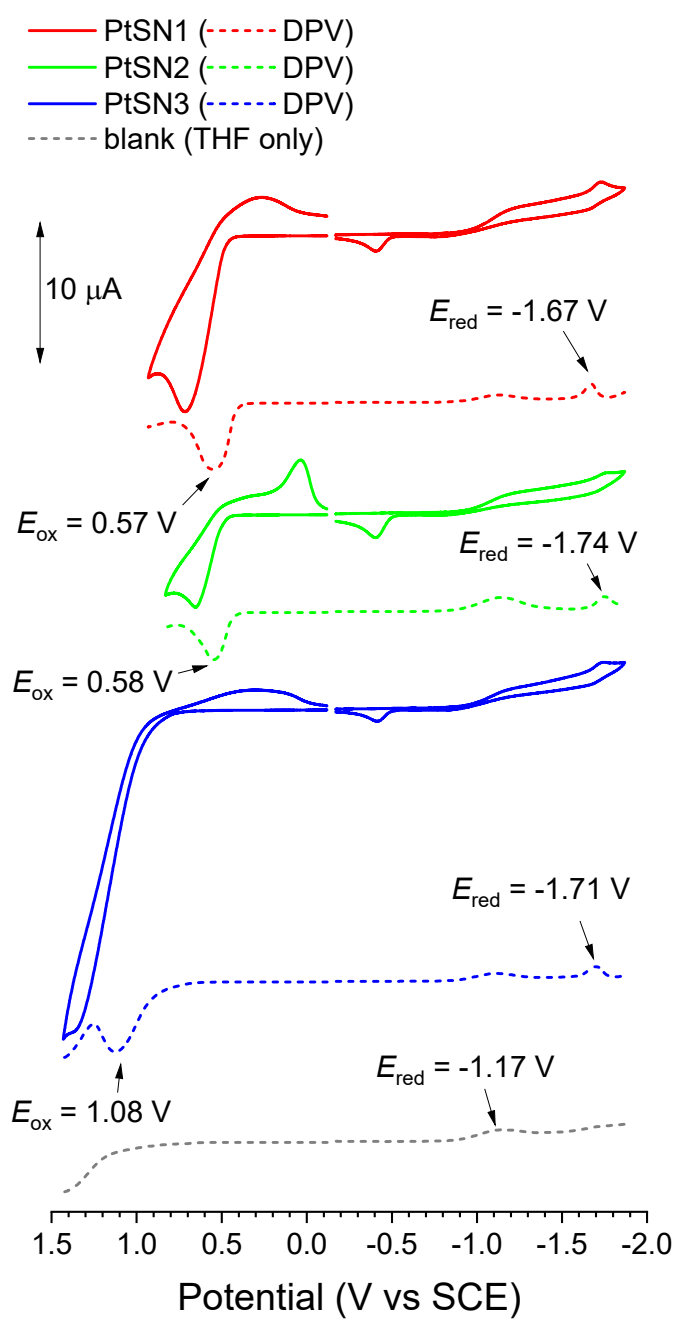

Figure S2. Cyclic (solid lines) and differential pulse (dashed lines) voltammograms of PtSN1 (red), PtSN2 (green) and PtSN3 (blue). $2.0 \mathrm{mM}$ PtSN was dissolved in Ar-saturated THF which contained 0.10 M TBAPF 6 supporting electrolyte. A Pt disc and a Pt wire were employed as the working and counter electrodes, respectively. $\mathrm{A} \mathrm{Ag} / \mathrm{AgNO}_{3}$ couple served as the pseudo reference electrode. Scan rate $=0.10 \mathrm{~V} \mathrm{~s}^{-1}$ (cyclic voltammetry) or $4 \mathrm{mV} \mathrm{s}^{-1}$ (differential pulse voltammetry). The $\mathrm{Fc} / \mathrm{Fc}^{+}$couple was employed as the external reference. A differential pulse voltammograms of a blank (i.e., Ar-saturated THF solution containing $0.10 \mathrm{M}$ TBAPF $_{6}$ ) was included for comparison. 

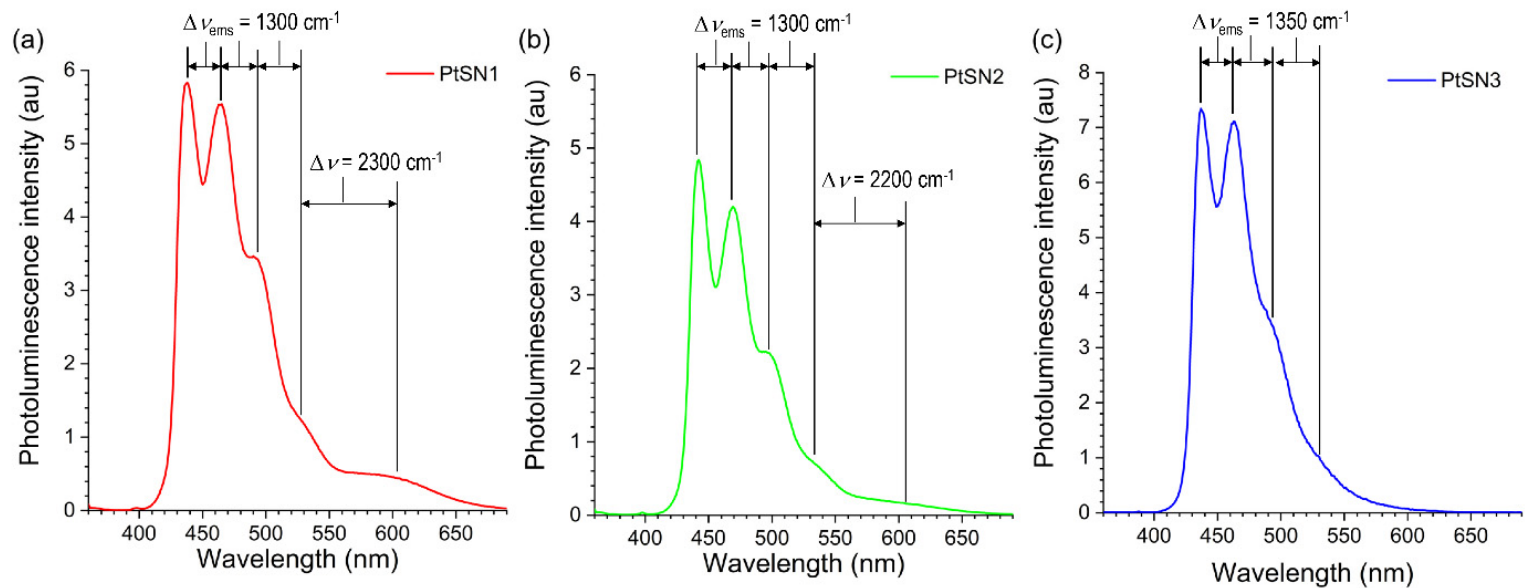

Figure S3. Vibronic progressions of the photoluminescence spectra ( $\Delta v_{\mathrm{ems}}$ ) of (a) PtSN1, (b) PtSN2 and (c) PtSN3 obtained in Ar-saturated toluene $(10 \mu \mathrm{M})$. The differences between the excimer emission bands and the fourth vibronic peaks are included for PtSN1-2. 
(a)

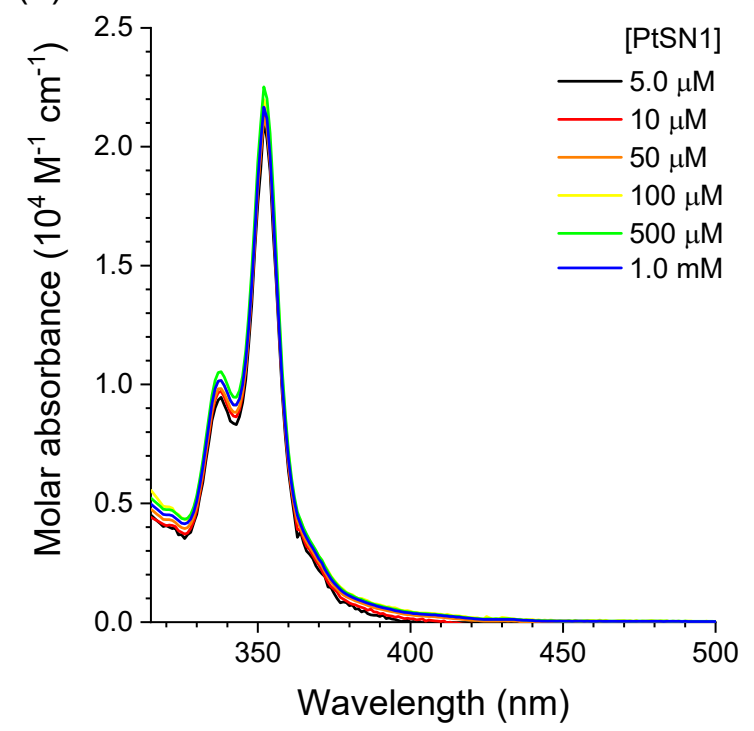

(b)

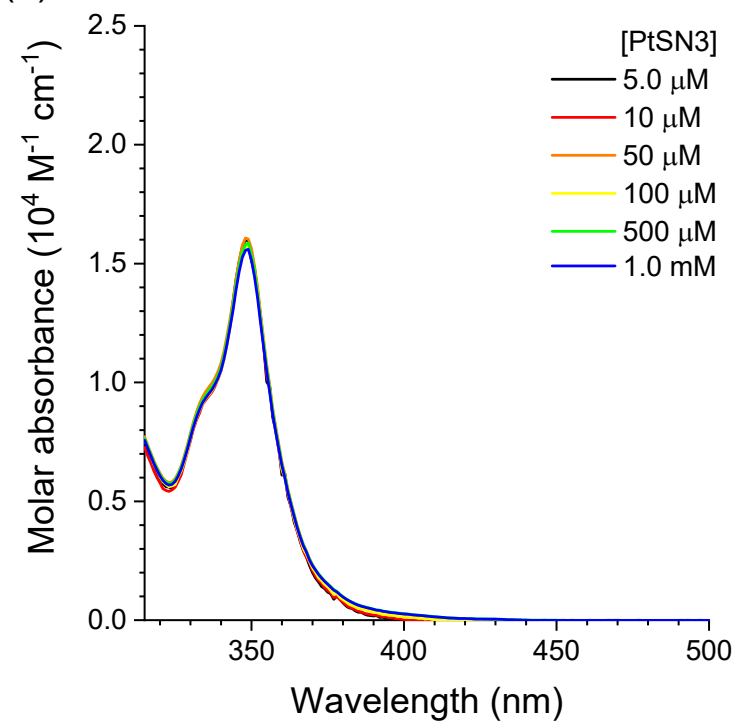

Figure S4. UV-vis absorption spectra of (a) PtSN1 and (b) PtSN3 recorded with increasing the concentration of the Pt complex (5.0 $\mu \mathrm{M}$ to $1.0 \mathrm{mM}$ in deaerated toluene). Note that the molar absorbance was plotted as a function of wavelength. 
(a)

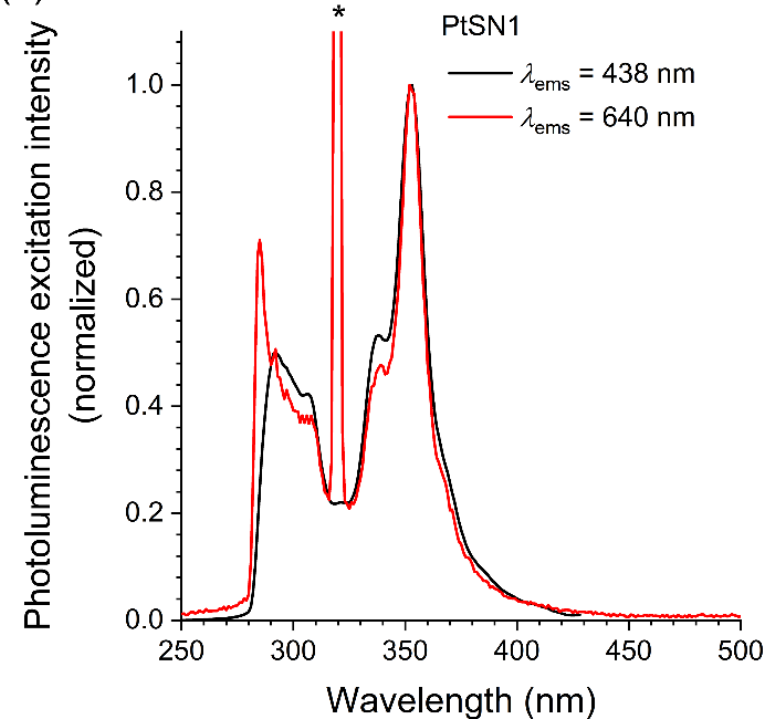

(b)

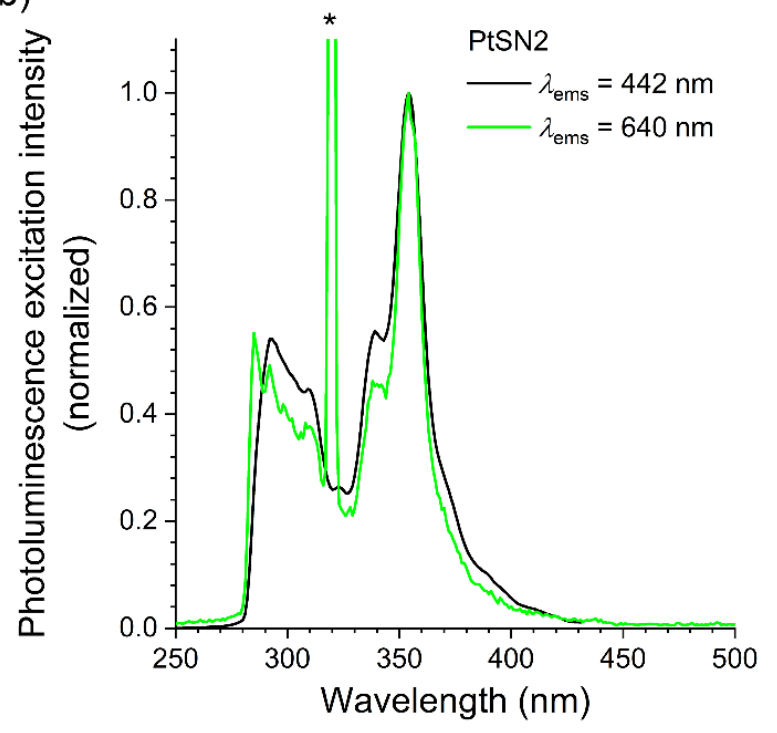

Figure S5. Photoluminescence excitation spectra of the monomer and excimer emissions of (a) PtSN1 and (b) PtSN2 recorded in deaerated toluene $(10 \mu \mathrm{M})$. Observation wavelengths $\left(\lambda_{\mathrm{ems}} \mathrm{s}\right)$ are indicated in the legend. The peaks marked with an asterisk $\left.{ }^{*}\right)$ were the overtone of $\lambda_{\text {ems }}(640 \mathrm{~nm})$. 
(a)

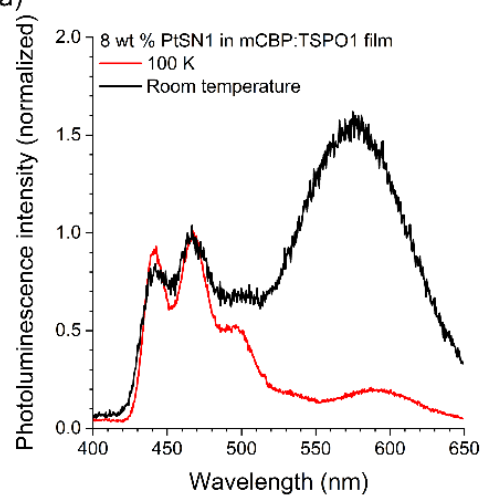

(b)

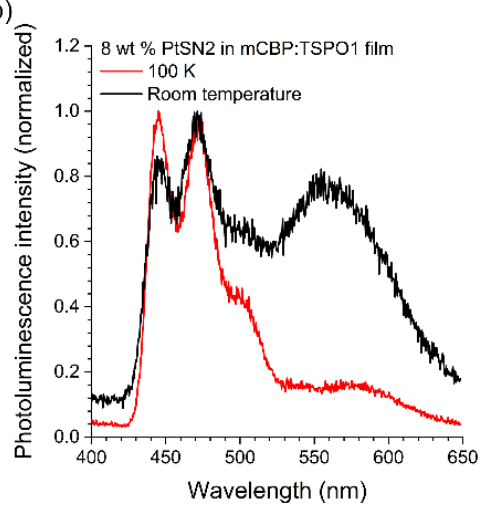

(c)

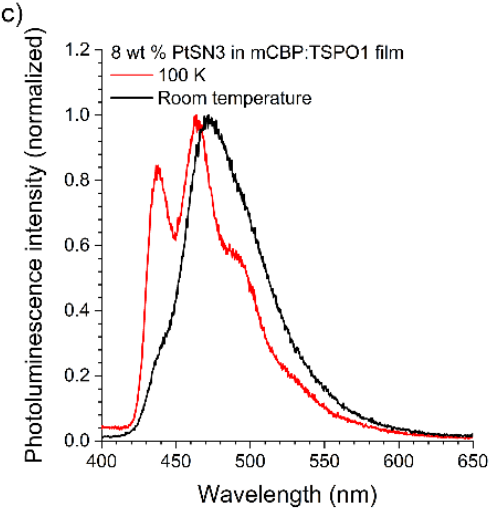

Figure S6. Comparisons of the photoluminescence spectra of mCBP:TSPO1 (1:1, wt/wt) films doped with $8 \mathrm{wt} \%$ PtSN dopants obtained at $100 \mathrm{~K}$ (red) and room temperature (black). (a) PtSN1, (b) PtSN2 and (c) PtSN3. 

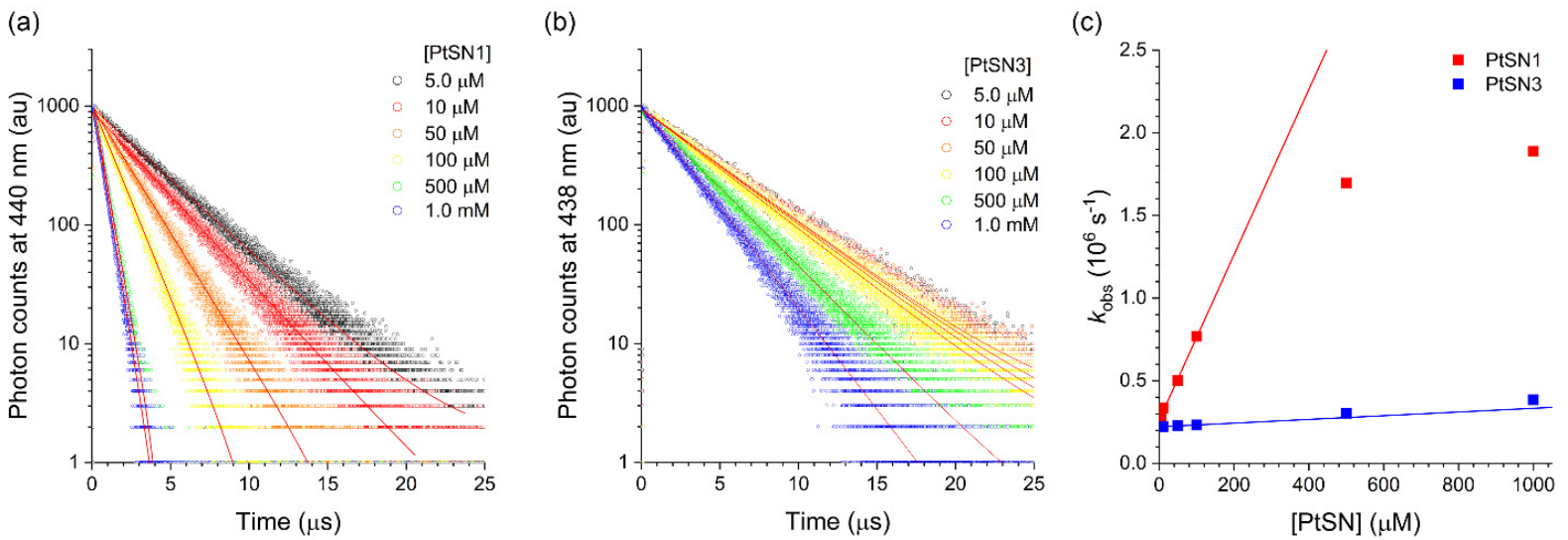

Figure S7. Photoluminescence decay traces of the monomer emissions of PtSN1 (a) and PtSN3 (b). (c) Plots of the observed decay rate ( $k_{\mathrm{obs}}, k_{\mathrm{obs}}=1 / \tau_{\mathrm{obs}} ; \tau_{\mathrm{obs}}$ is the time constant of the monoexponential decay fit of the photoluminescence decay trace) as a function of the concentration of PtSN1 (red squares) and PtSN3 (blue squares). The lines are fit to an equation, $k_{\mathrm{obs}}=k_{\mathrm{M}}+k_{1} \cdot[\mathrm{PtSN}]$. In this equation, $k_{\mathrm{M}}$ and $k_{1}$ are the intrinsic decay rate of a monomer and the self-quenching rate due to intermolecular interactions, respectively. Fit results are $k_{\mathrm{M}}(\mathrm{PtSN} 1)=2.6 \times 10^{5} \mathrm{~s}^{-1}, \mathrm{~km}_{\mathrm{M}}(\mathrm{PtSN} 3)=2.2 \times 10^{5} \mathrm{~s}^{-1}, \mathrm{k}_{1}(\mathrm{PtSN} 1)=5.0 \times 10^{9} \mathrm{M}^{-1}$ $\mathrm{s}^{-1}$, and $k_{1}(\mathrm{PtSN} 3)=0.11 \times 10^{9} \mathrm{M}^{-1} \mathrm{~s}^{-1}$. 
(a)

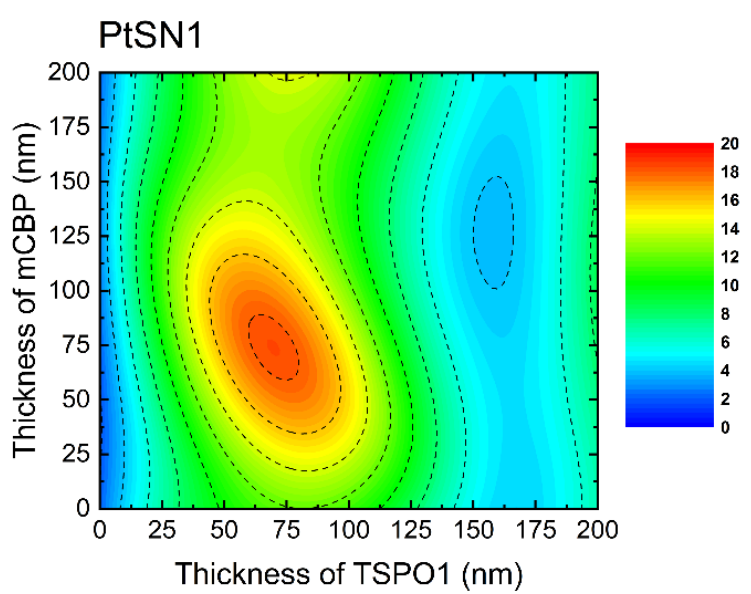

(b)

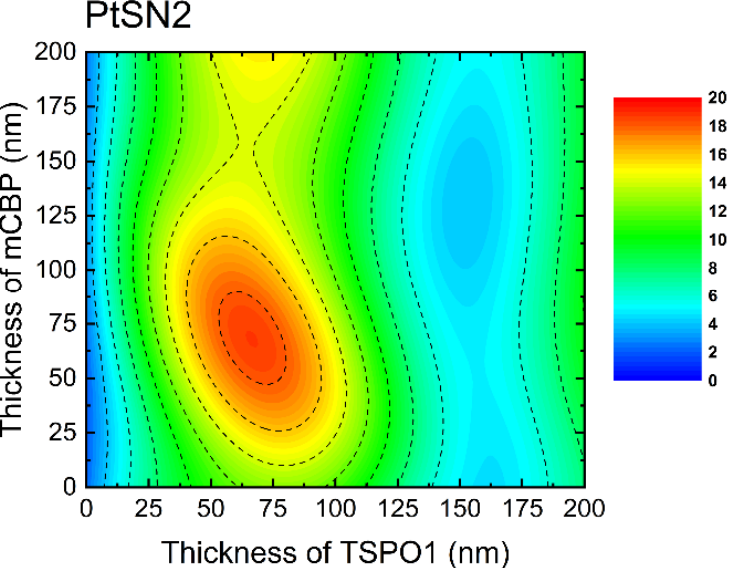

(c)

\begin{abstract}
PtSN3
\end{abstract}

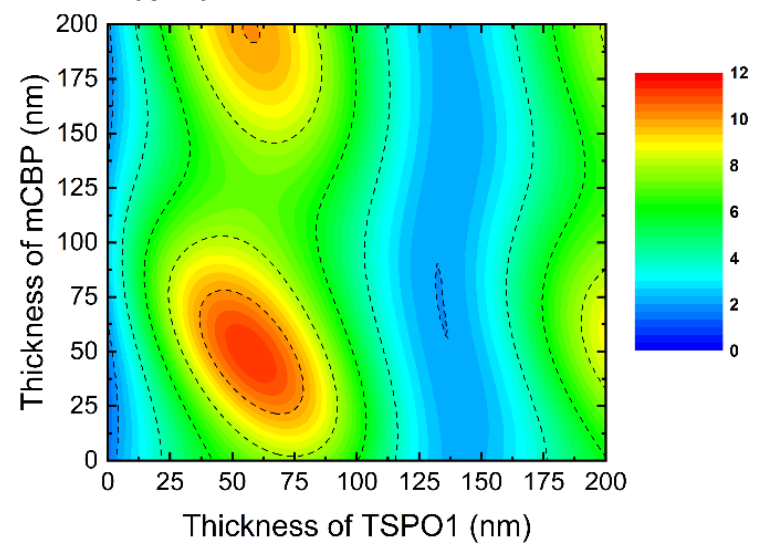

Figure S8. Simulated external quantum efficiencies (EQEs) of a device with a configuration of ITO (70 $\mathrm{nm}) / \mathrm{mCBP}: 6$ wt $\% \operatorname{Re}_{2} \mathrm{O}_{7}$ (y nm)/mCBP $(10 \mathrm{~nm}) / \mathrm{mCBP}: T S P 01: 8$ wt \% dopant $(20 \mathrm{~nm}) / \mathrm{TSPO} 1$ (x $\mathrm{nm}) / \mathrm{Mg}$ :Liq $(2 \mathrm{~nm}) / \mathrm{Al}(100 \mathrm{~nm})$. The EQEs were simulated with varying the $\mathrm{x}$ and $\mathrm{y}$ values. 
(a)

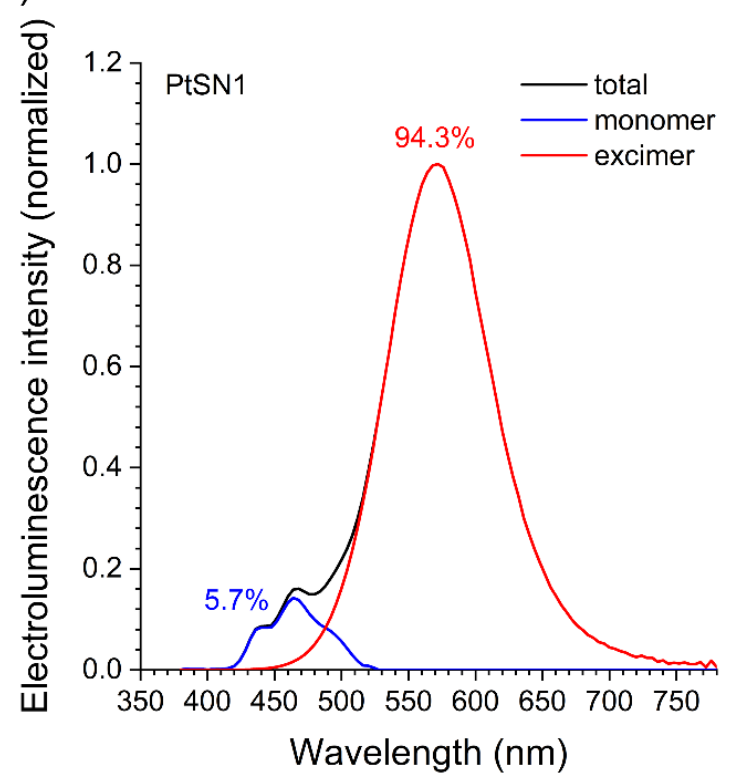

(b)

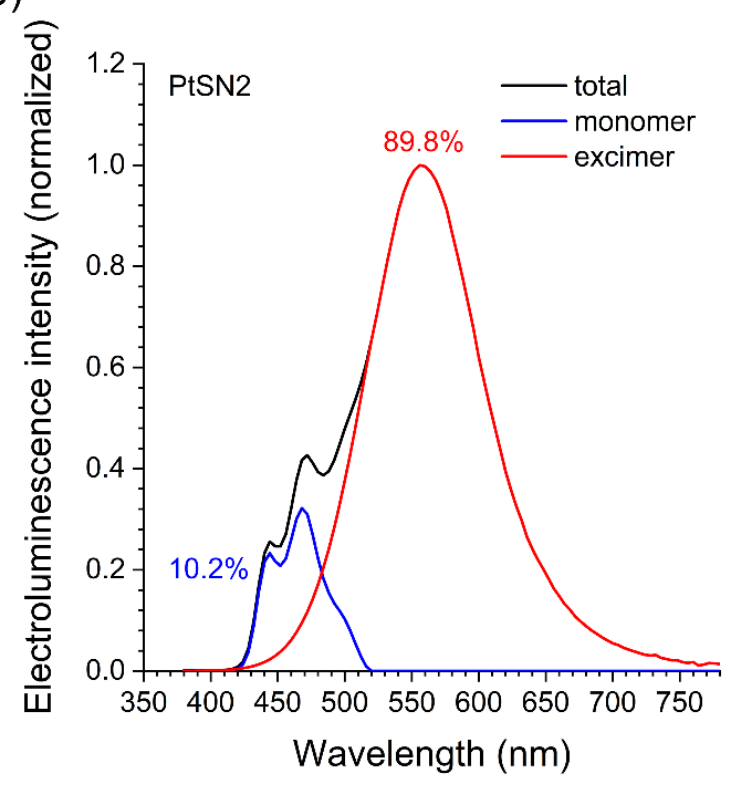

Figure S9. Deconvolution of the electroluminescence spectra of the devices of (a) PtSN1 and (d) PtSN2 recorded at $100 \mathrm{~cd} \mathrm{~m}^{-2}$. The black total spectra were deconvoluted into a monomer (blue) and an excimer (red) emissions. The numbers are the relative integrated values of the deconvoluted spectra. 
(a)

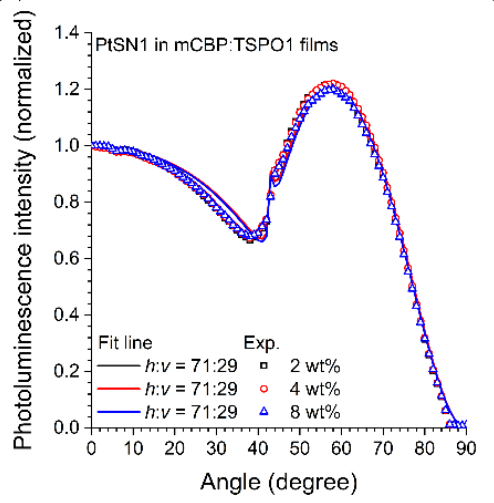

(b)

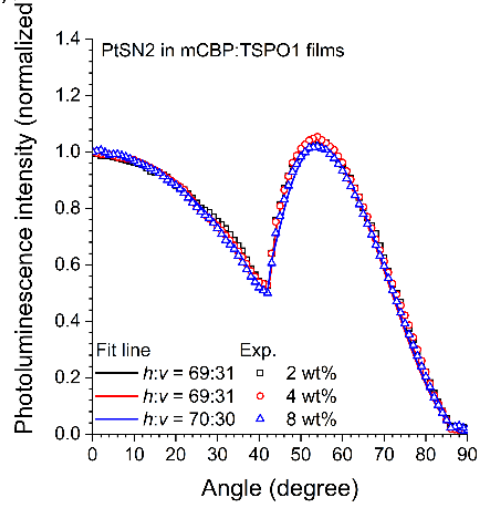

(c)

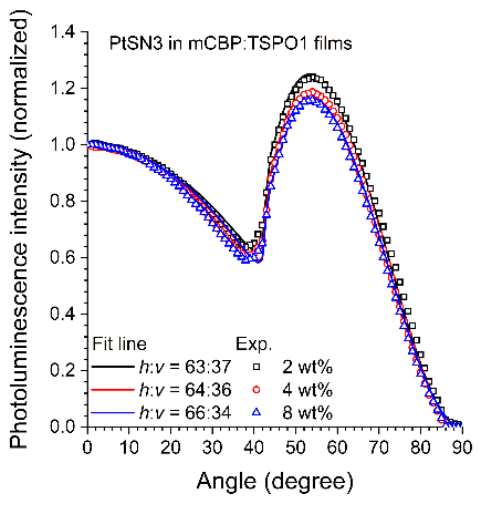

Figure S10. Angle-dependent photoluminescence intensities of the p-polarized lights from mCBP:TSPO1 films doped with 2, 4 and 8 wt \% PtSN dopants. (a) PtSN1, (b) PtSN2 and (c) PtSN3. The solid curves were constructed with horizontal orientation values of the electric transition dipole moment $(h /(h+v) ; h$ and $v$ refer to the magnitudes of the horizontal and vertical emitting dipoles) included in the legends. 

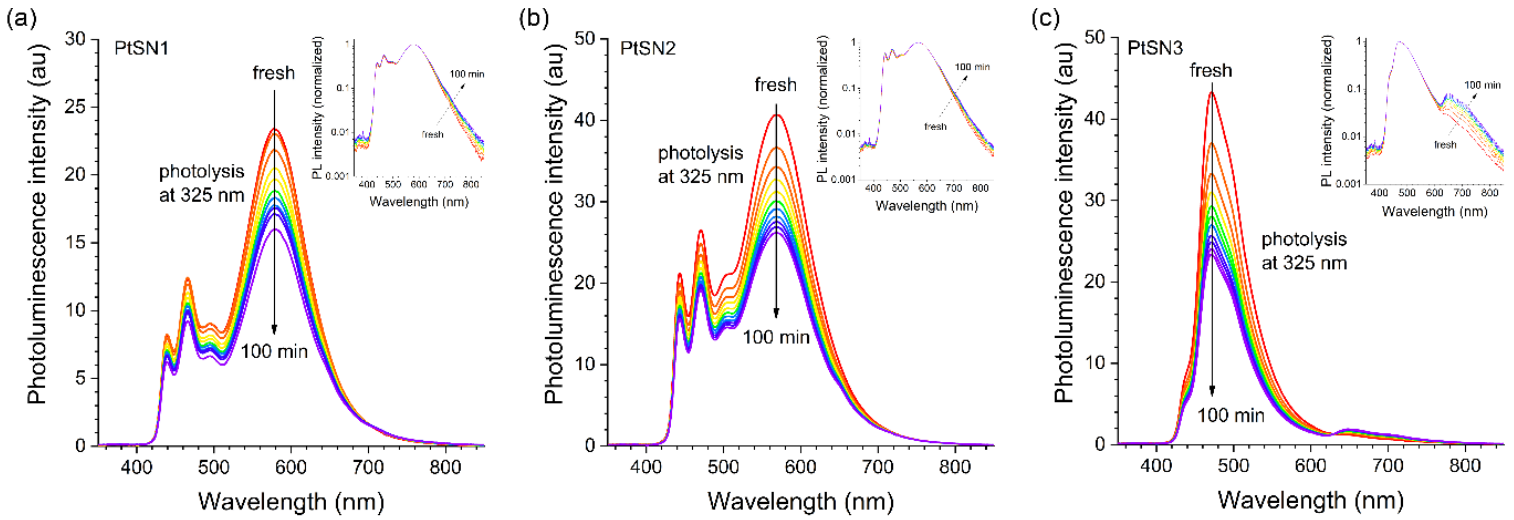

Figure S11. Photoluminescence spectra of the mCBP:TSPO1 (1:1, wt/wt) thin films doped with 8 wt \% PtSN dopants recorded during continuous photoillumination at $325 \mathrm{~nm}$ using a $5 \mathrm{~mW}$ He:Cd laser for 100 min. (a) PtSN1, (b) PtSN2 and (c) PtSN3. The inset graphs are normalized spectra. 
(a)

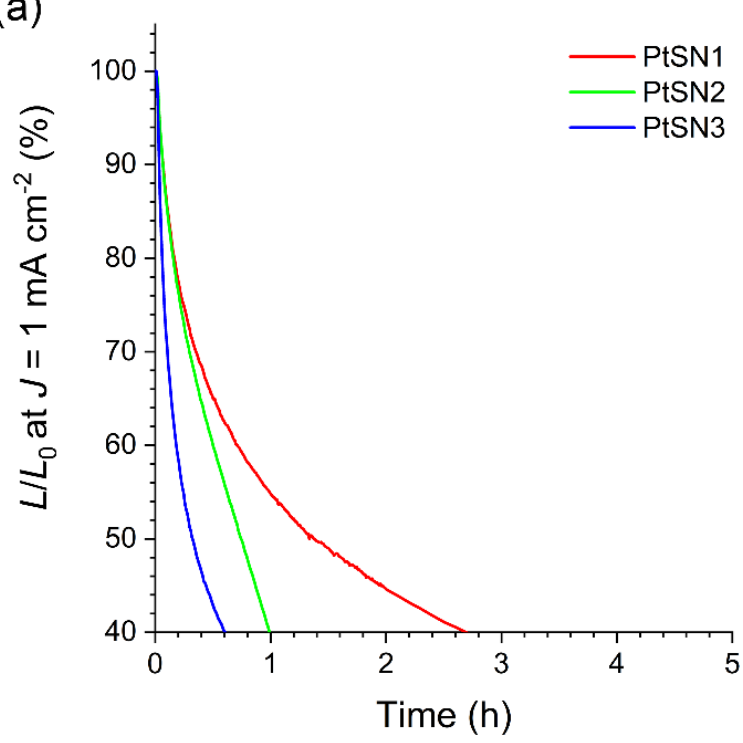

(b)

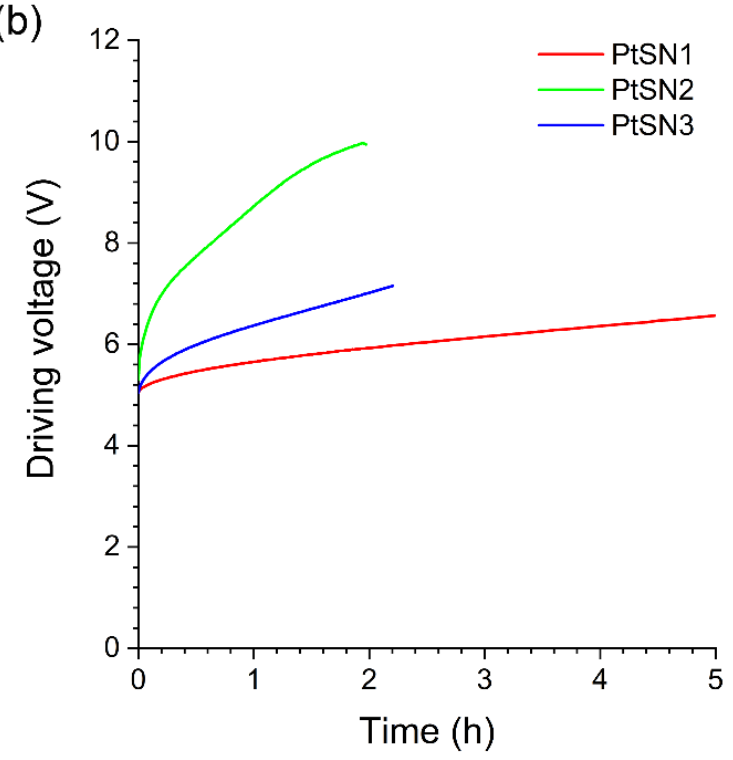

Figure S12. (a) Relative luminance $\left(L / L_{0}\right)$ and (b) driving voltage as a function of operation time of the PtSN devices driven at a constant current density of an initial value of $1 \mathrm{~mA} \mathrm{~cm}-2$. 


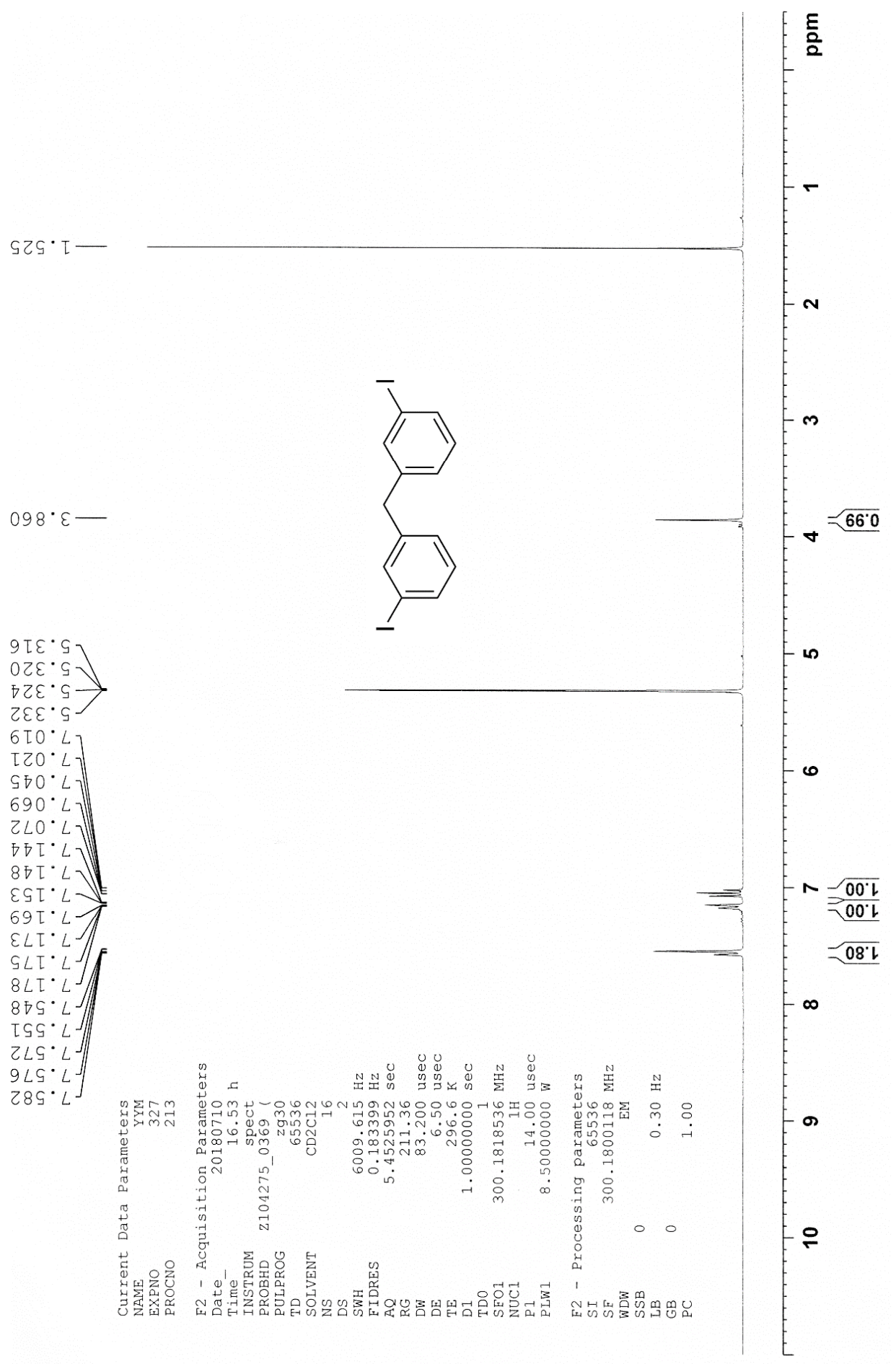

Figure S13. ${ }^{1} \mathrm{H}$ NMR spectrum $\left(300 \mathrm{MHz}, \mathrm{CD}_{2} \mathrm{Cl}_{2}\right)$ of di(3-iodophenyl)methane. 

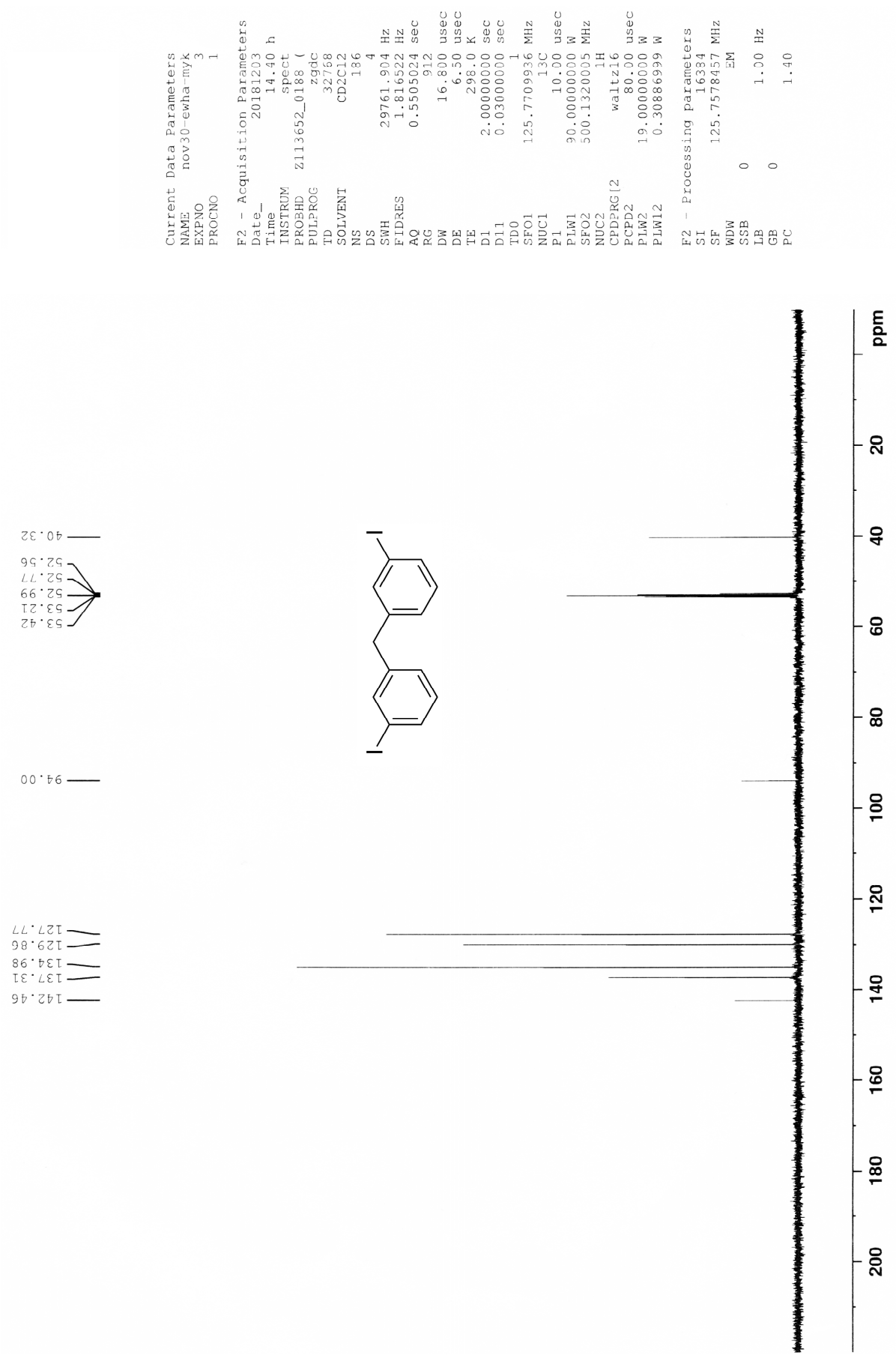

Figure S14. ${ }^{13} \mathrm{C}\left\{{ }^{1} \mathrm{H}\right\}$ NMR spectrum (126 MHz, $\left.\mathrm{CD}_{2} \mathrm{Cl}_{2}\right)$ of di(3-iodophenyl)methane. 


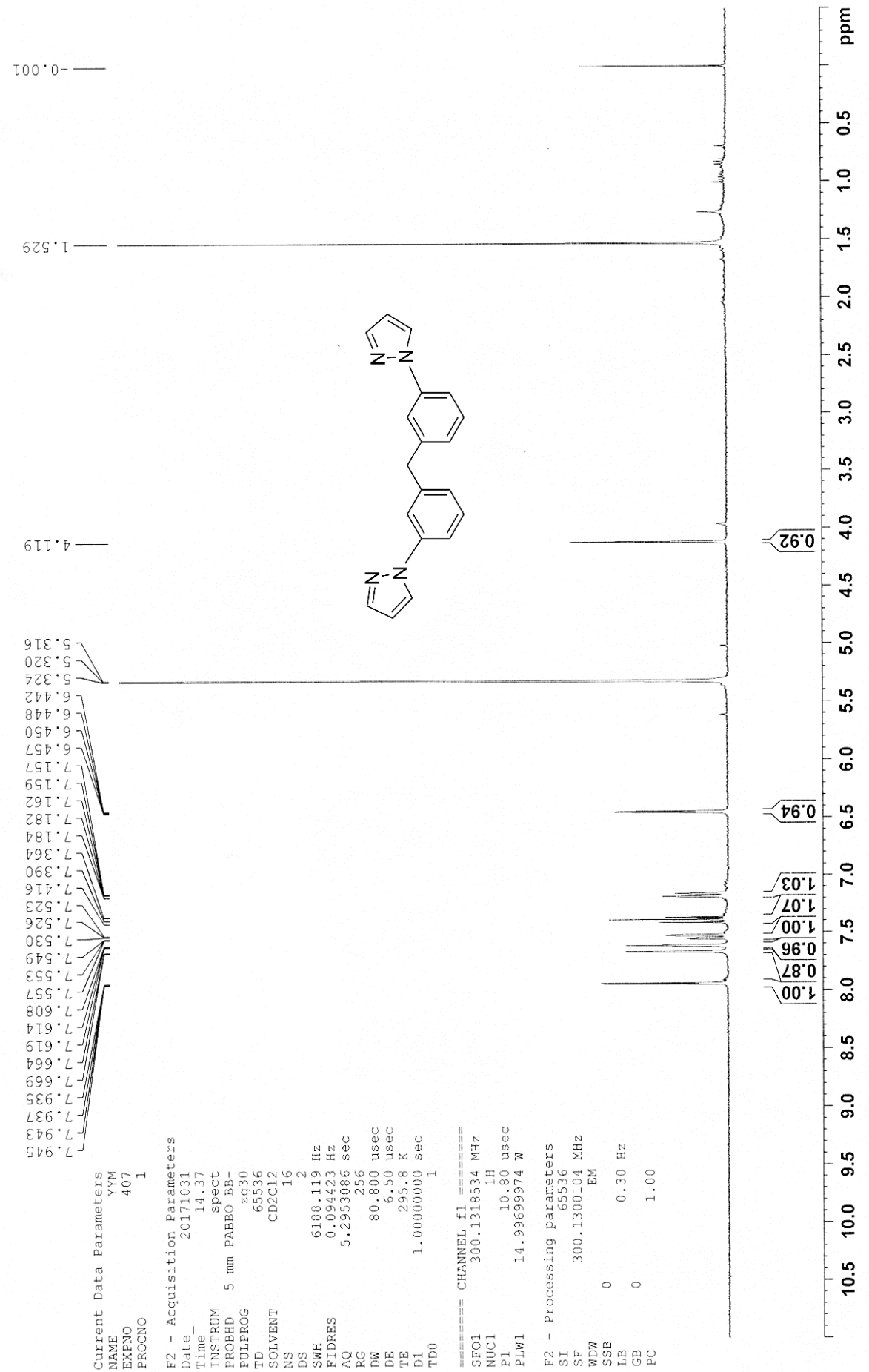

Figure S15. ${ }^{1} \mathrm{H}$ NMR spectrum $\left(300 \mathrm{MHz}, \mathrm{CD}_{2} \mathrm{Cl}_{2}\right)$ of $\mathrm{SN} 1 \mathrm{H}_{2}$. 

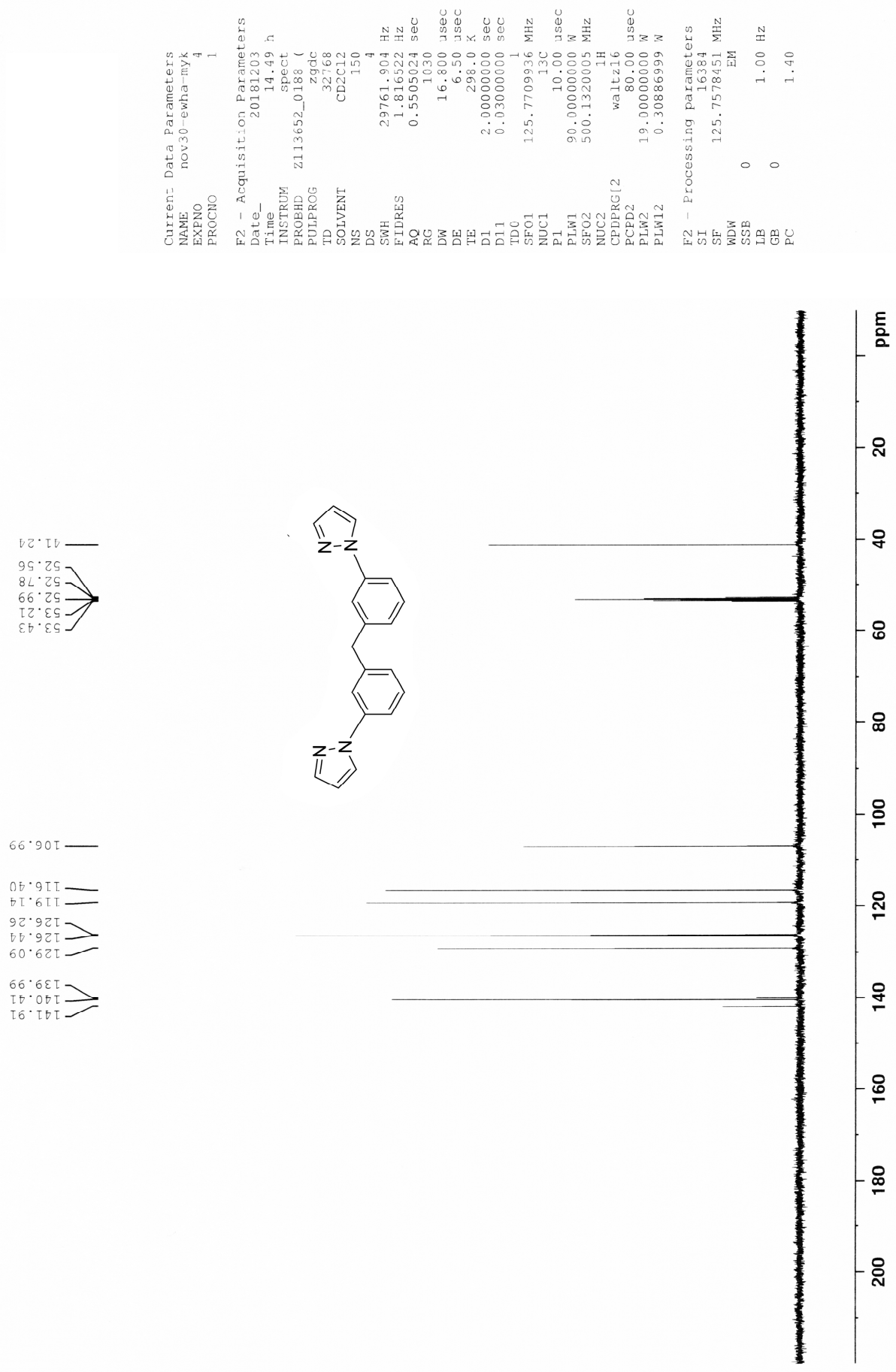

Figure S16. ${ }^{13} \mathrm{C}\left\{{ }^{1} \mathrm{H}\right\} \mathrm{NMR}$ spectrum $\left(126 \mathrm{MHz}, \mathrm{CD}_{2} \mathrm{Cl}_{2}\right)$ of $\mathrm{SN} 1 \mathrm{H}_{2}$. 


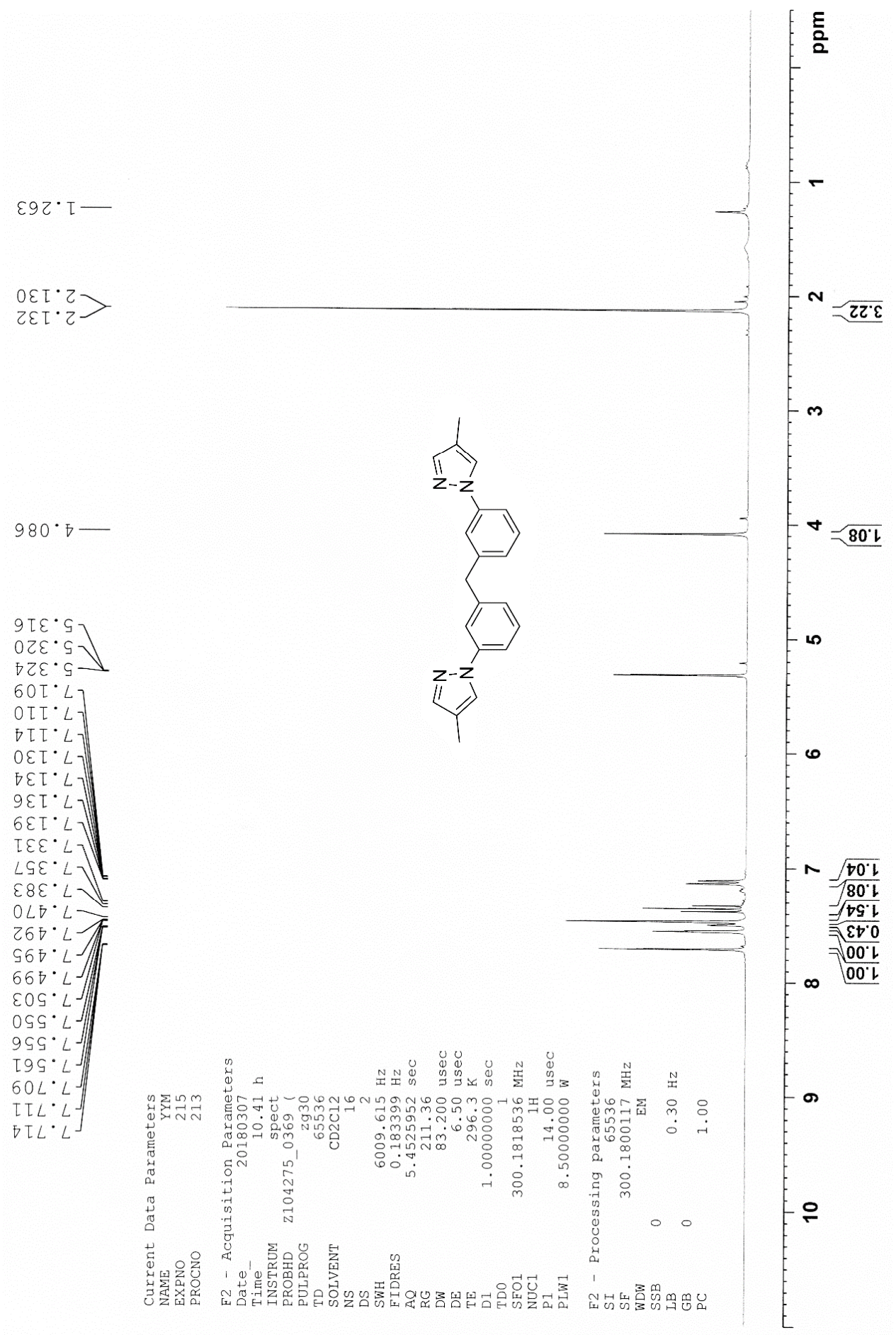

Figure S17. ${ }^{1} \mathrm{H}$ NMR spectrum $\left(300 \mathrm{MHz}, \mathrm{CD}_{2} \mathrm{Cl}_{2}\right)$ of $\mathrm{SN}_{2} \mathrm{H}_{2}$. 

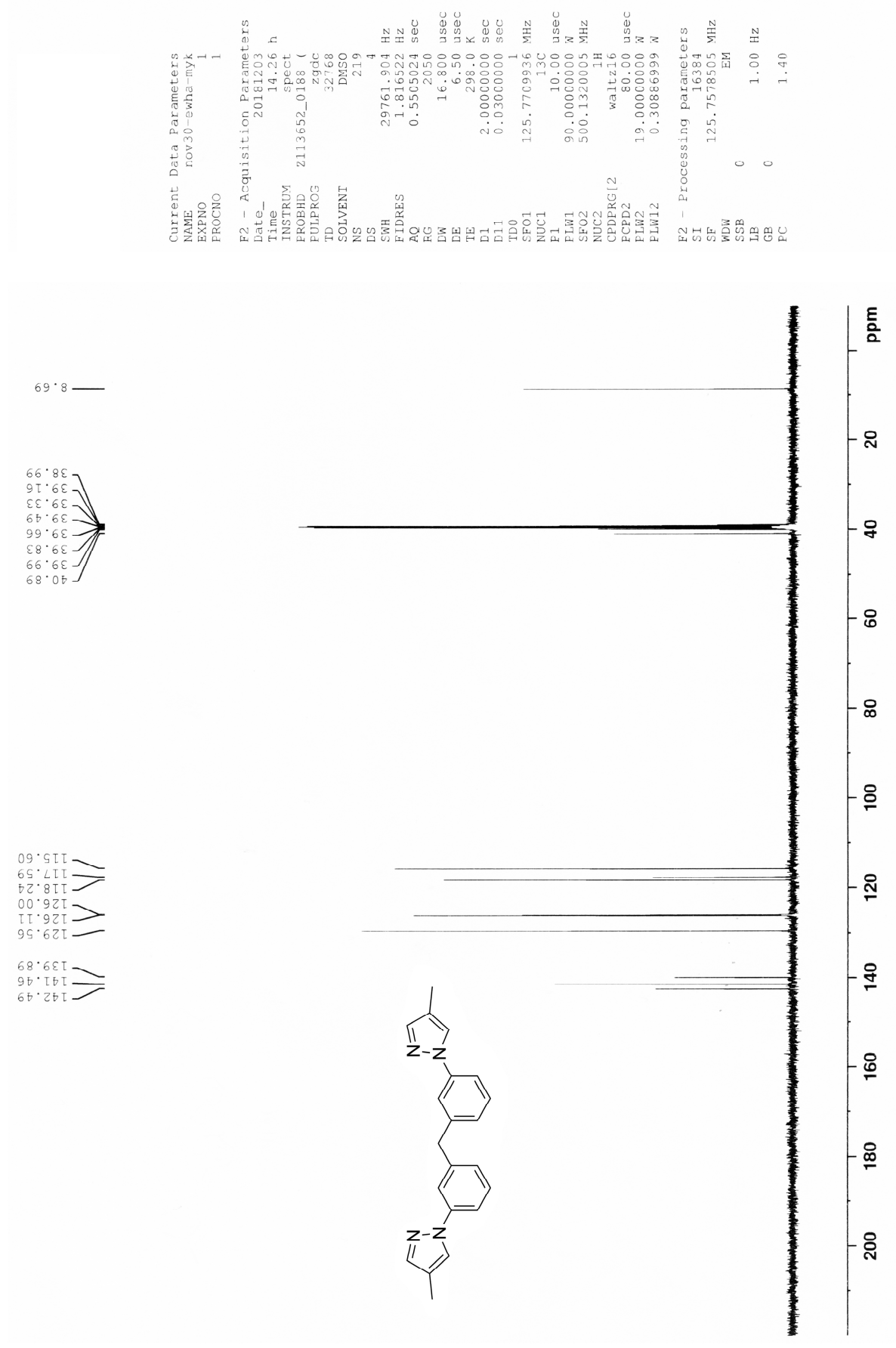

Figure S18. ${ }^{13} \mathrm{C}\left\{{ }^{1} \mathrm{H}\right\}$ NMR spectrum (126 MHz, DMSO-d $)$ of $\mathrm{SN}_{2} \mathrm{H}_{2}$. 


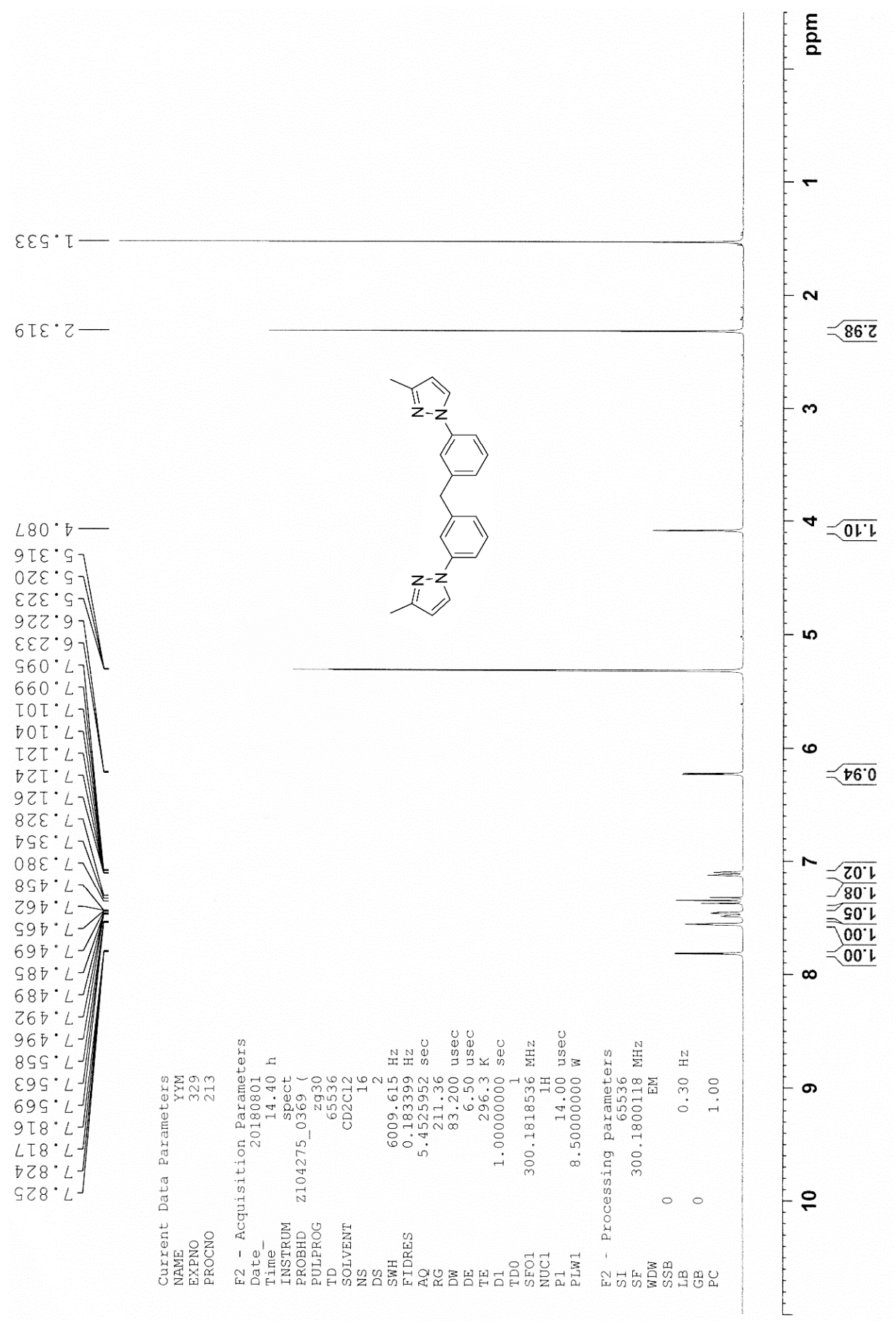

Figure S19. ${ }^{1} \mathrm{H}$ NMR spectrum $\left(300 \mathrm{MHz}, \mathrm{CD}_{2} \mathrm{Cl}_{2}\right)$ of $\mathrm{SN}_{3} \mathrm{H}_{2}$. 

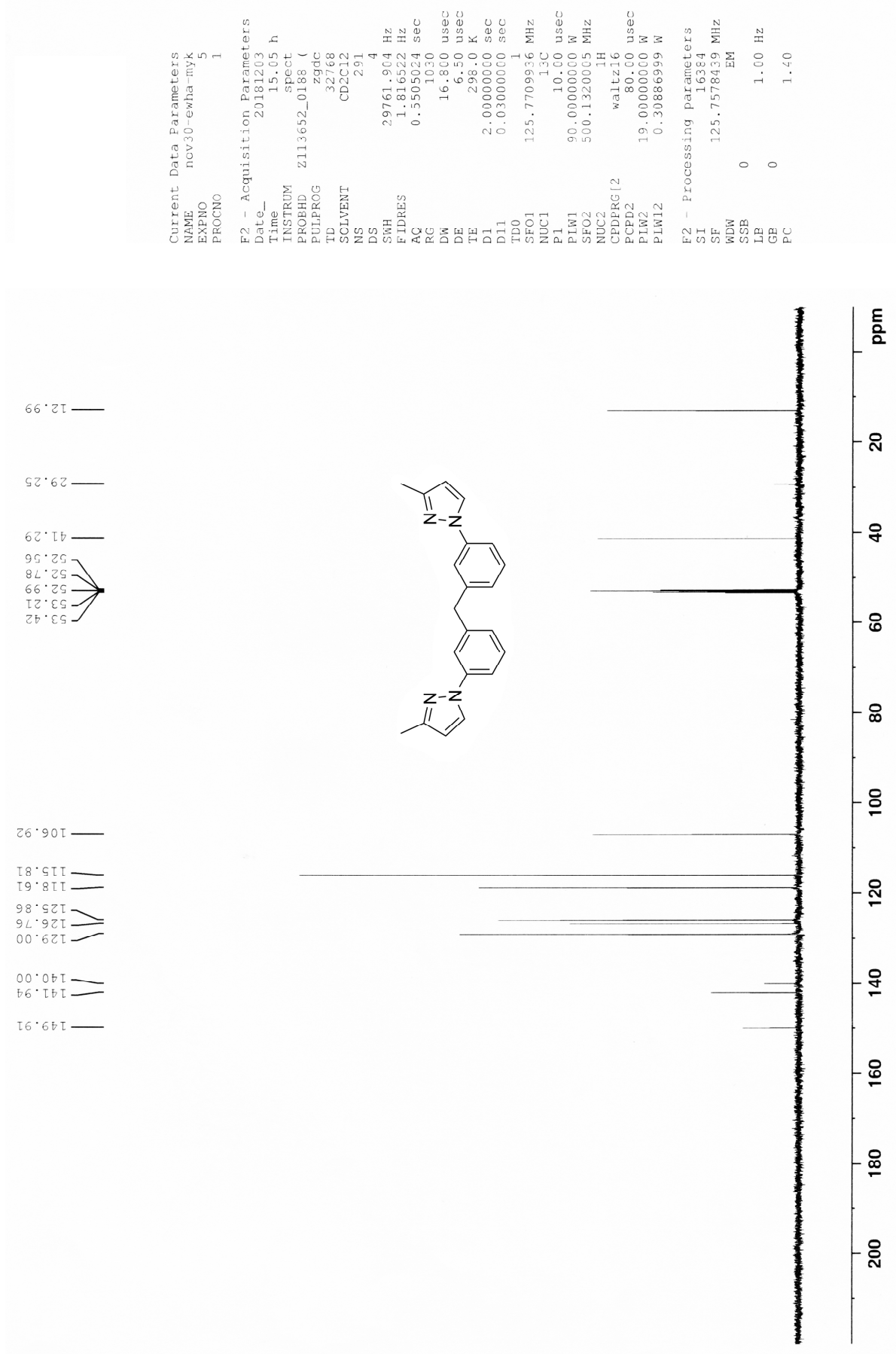

Figure S20. ${ }^{13} \mathrm{C}\left\{{ }^{1} \mathrm{H}\right\}$ NMR spectrum $\left(126 \mathrm{MHz}, \mathrm{CD}_{2} \mathrm{Cl}_{2}\right)$ of $\mathrm{SN}_{3} \mathrm{H}_{2}$. 


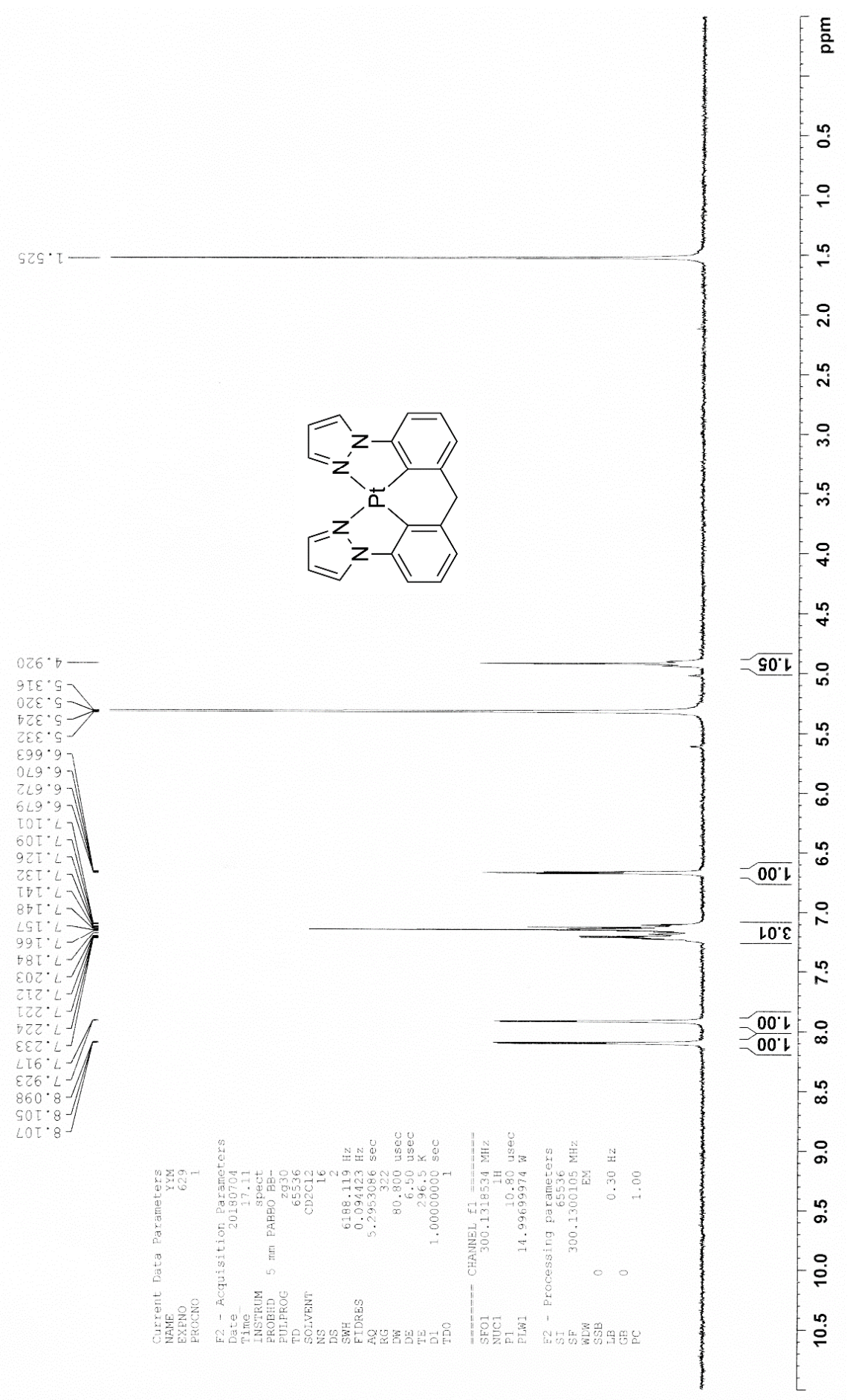

Figure S21. ${ }^{1} \mathrm{H}$ NMR spectrum $\left(300 \mathrm{MHz}, \mathrm{CD}_{2} \mathrm{Cl}_{2}\right)$ of PtSN1. 

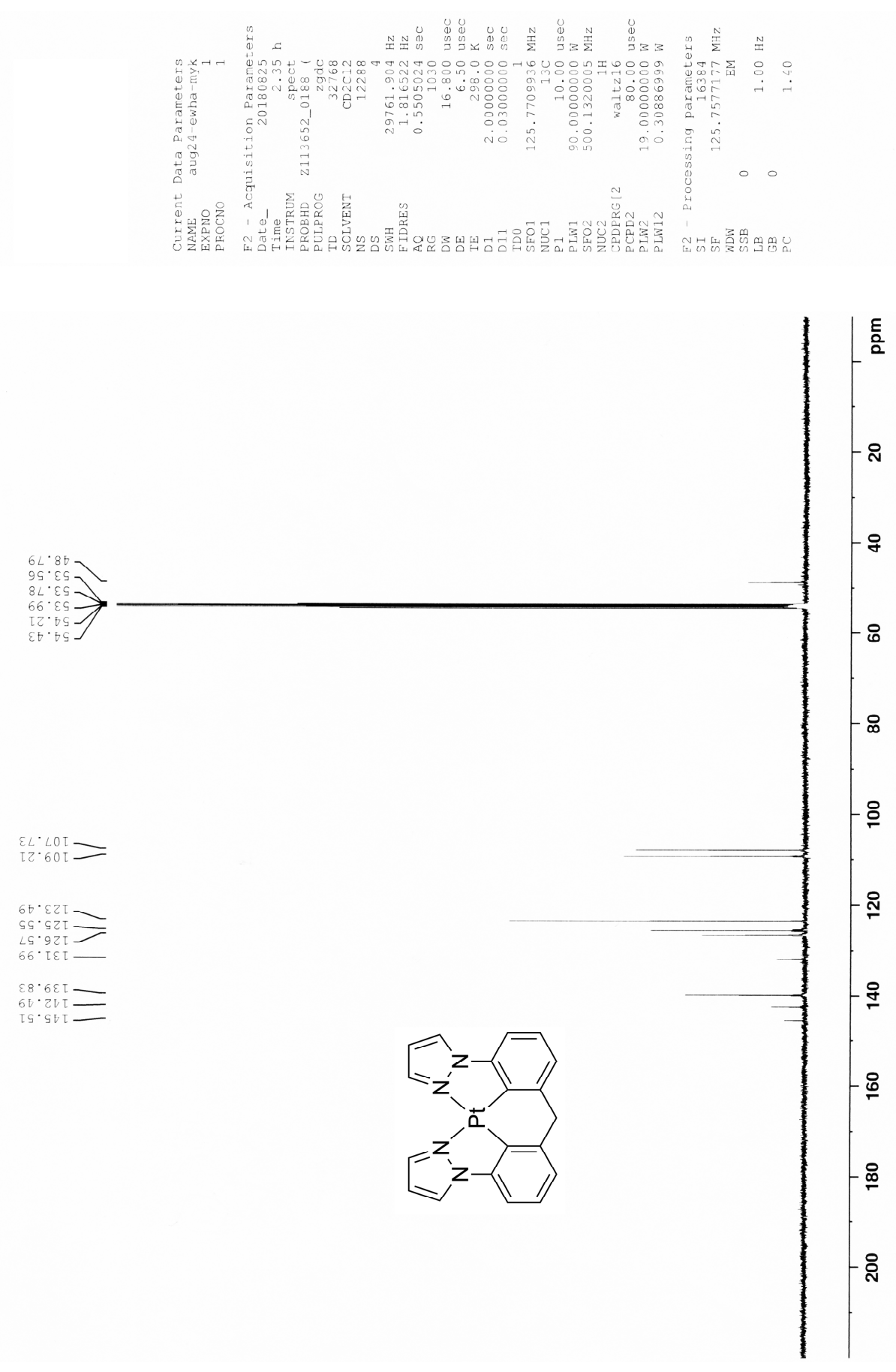

Figure S22. ${ }^{13} \mathrm{C}\left\{{ }^{1} \mathrm{H}\right\}$ NMR spectrum $\left(126 \mathrm{MHz}, \mathrm{CD}_{2} \mathrm{Cl}_{2}\right)$ of PtSN1. 


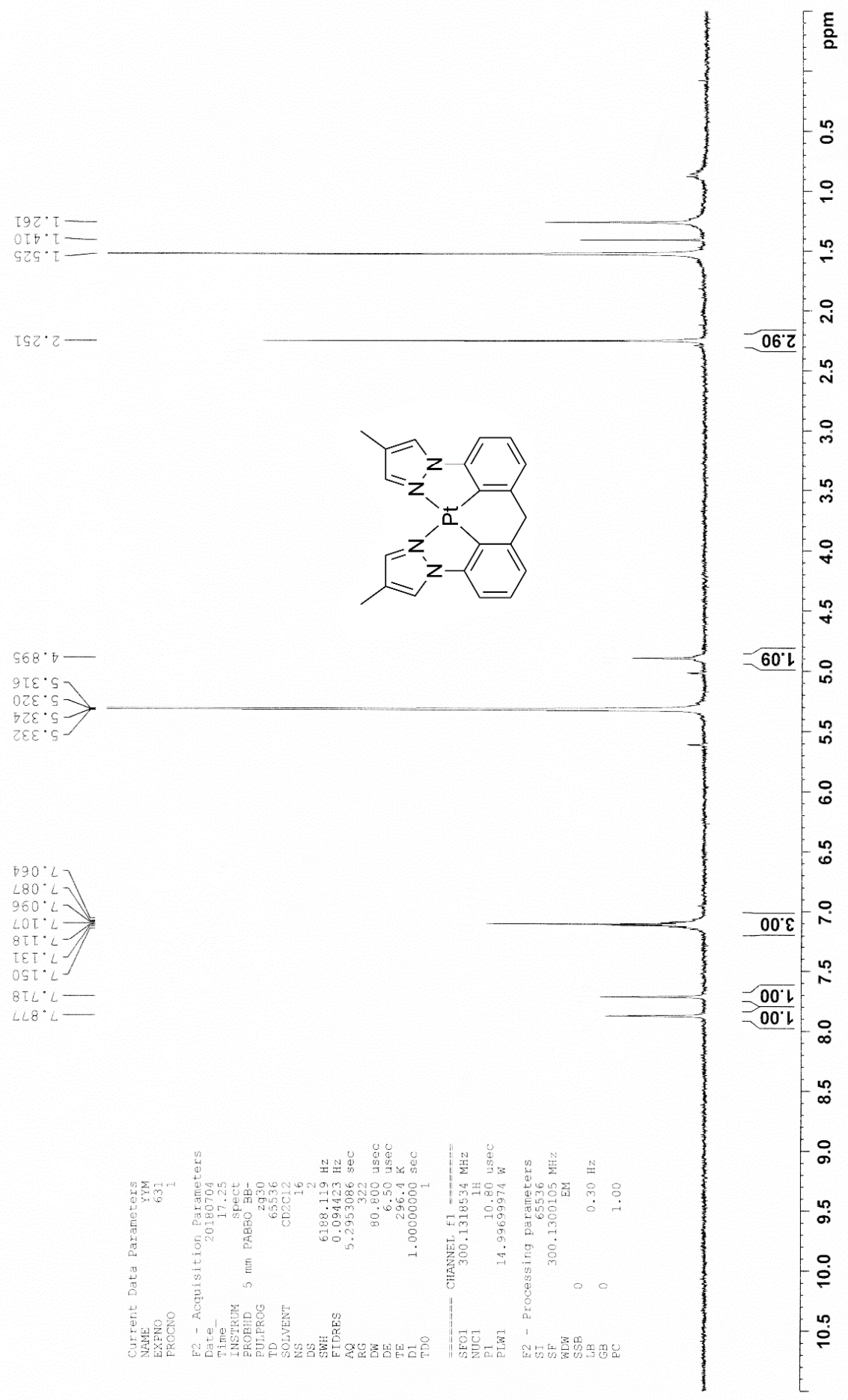

Figure S23. ${ }^{1} \mathrm{H}$ NMR spectrum $\left(300 \mathrm{MHz}, \mathrm{CD}_{2} \mathrm{Cl}_{2}\right)$ of PtSN2. 

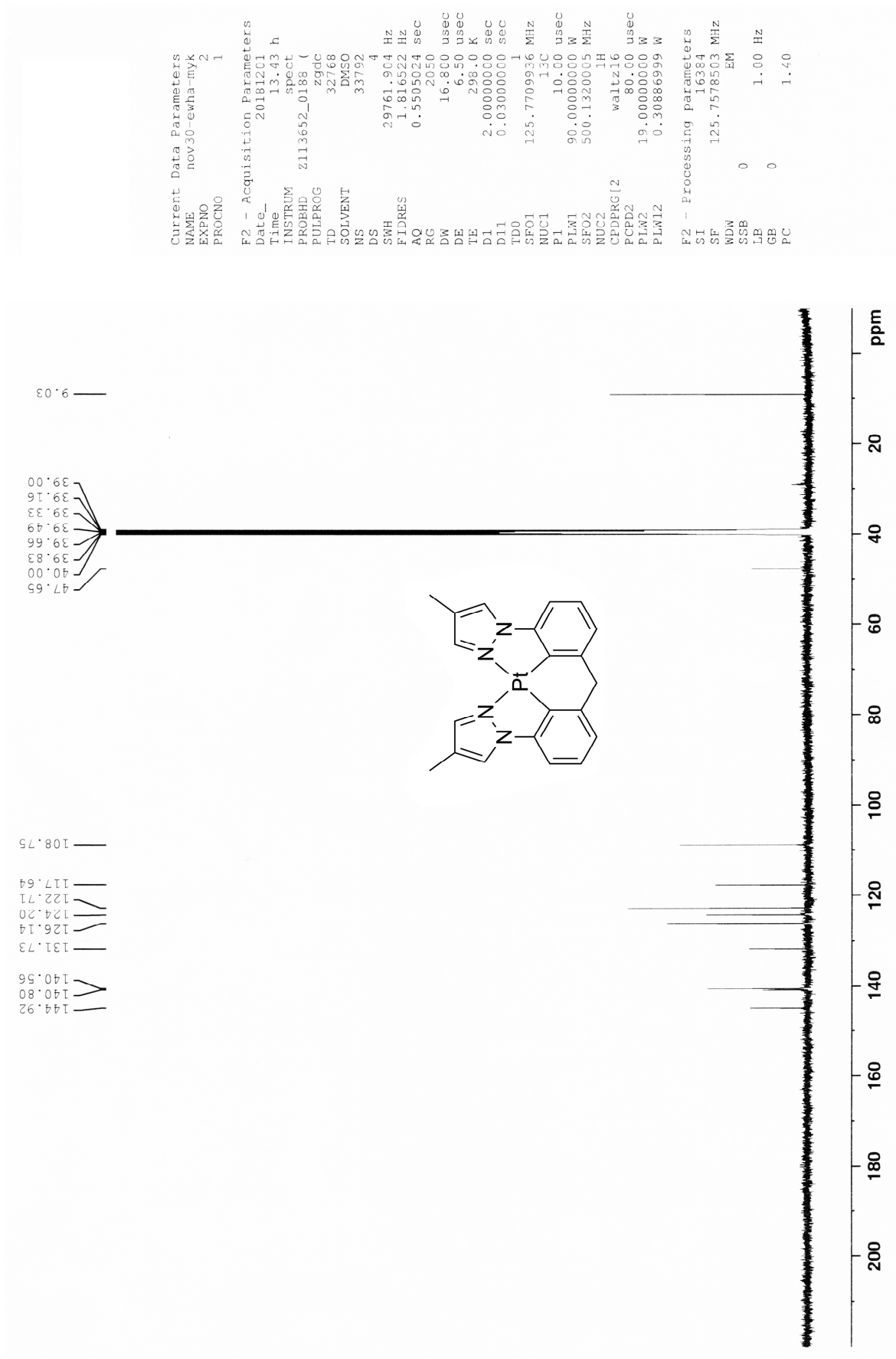

Figure S24. ${ }^{13} \mathrm{C}\left\{{ }^{1} \mathrm{H}\right\}$ NMR spectrum (126 MHz, DMSO-d $)$ of PtSN2. 


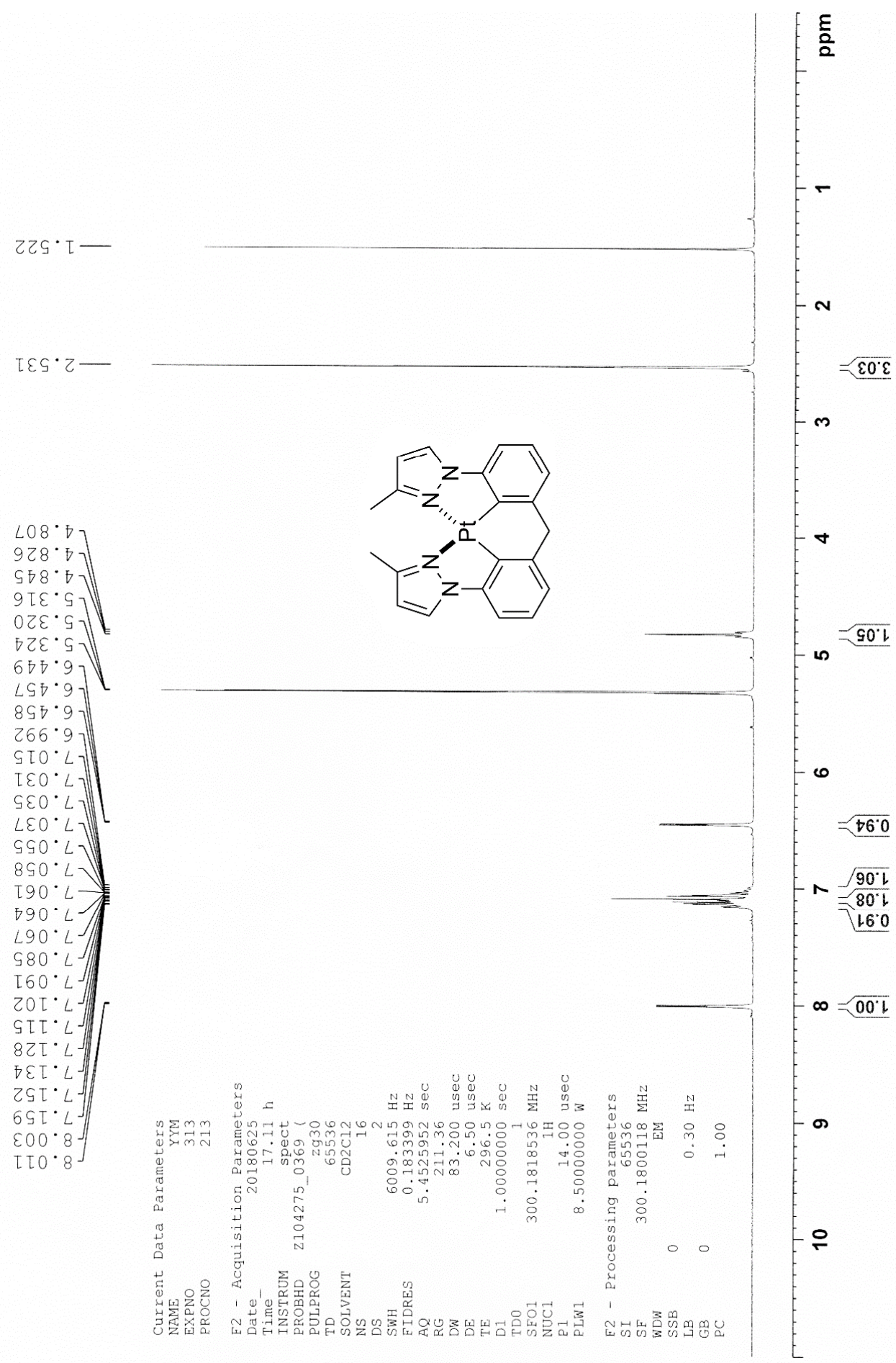

Figure S25. ${ }^{1} \mathrm{H}$ NMR spectrum $\left(300 \mathrm{MHz}, \mathrm{CD}_{2} \mathrm{Cl}_{2}\right)$ of PtSN3. 

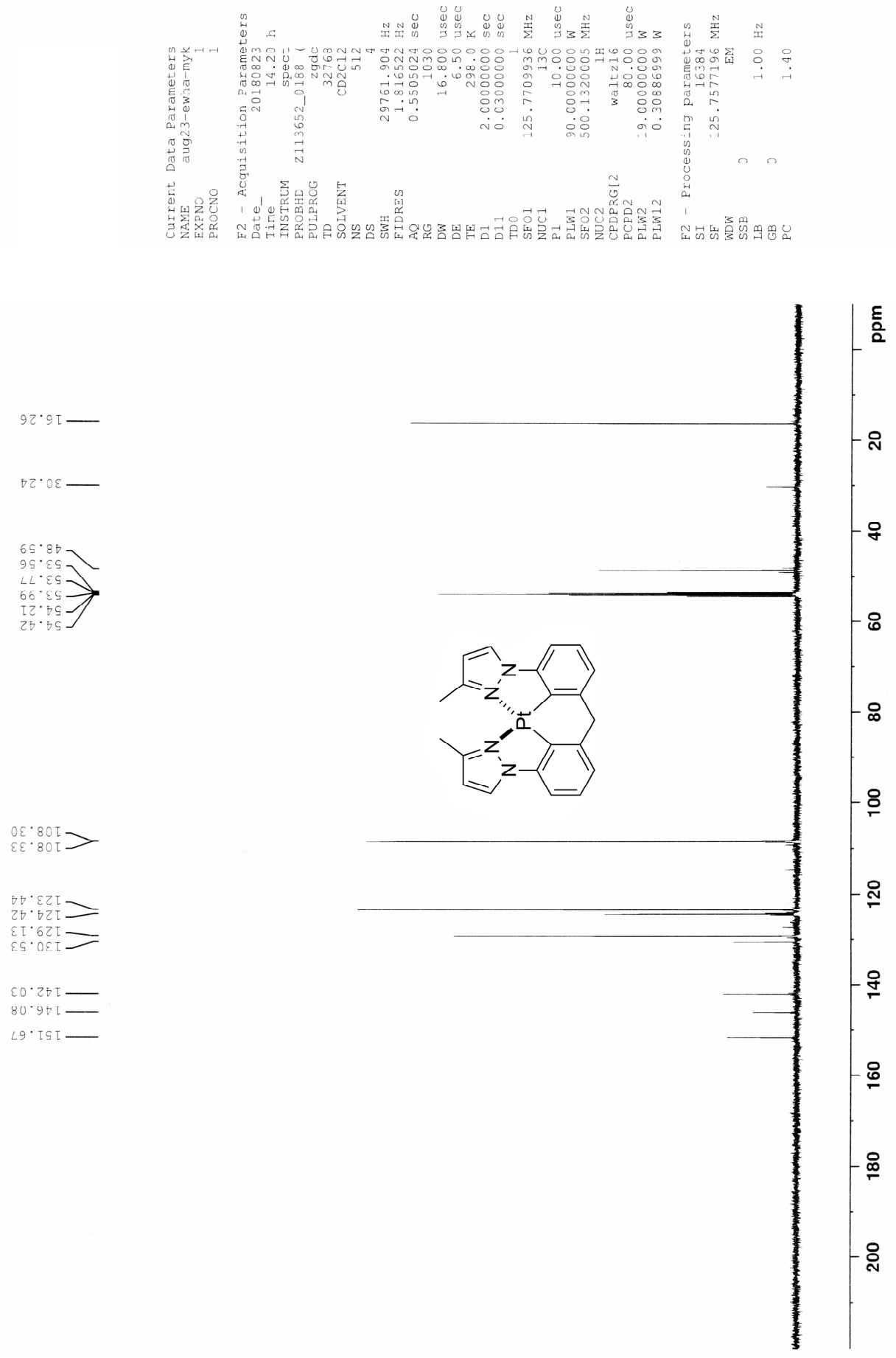

Figure S26. ${ }^{13} \mathrm{C}\left\{{ }^{1} \mathrm{H}\right\}$ NMR spectrum (126 MHz, $\mathrm{CD}_{2} \mathrm{Cl}_{2}$ ) of PtSN3. 
Table S1. TD-CAM-B3LYP Calculation Results for PtSN1

\begin{tabular}{ccc}
\hline state & energy $(\mathrm{eV})$ & $\begin{array}{c}\text { participating molecular orbitals } \\
\text { (expansion coefficient) }\end{array}$ \\
\hline $\mathrm{T}_{1}$ & 2.97 & HOMO-1 $\rightarrow$ LUMO $(0.61)$ \\
\hline $\mathrm{T}_{2}$ & 2.97 & $\mathrm{HOMO} \rightarrow$ LUMO $(0.61)$ \\
\hline $\mathrm{S}_{1}$ & 3.24 & $\mathrm{HOMO} \rightarrow$ LUMO $(0.70)$ \\
$\mathrm{T}_{3}$ & 3.27 & $\mathrm{HOMO}-3 \rightarrow$ LUMO $(0.47)$ \\
$\mathrm{T}_{4}$ & 3.44 & HOMO $-2 \rightarrow$ LUMO $(0.70)$ \\
\hline
\end{tabular}

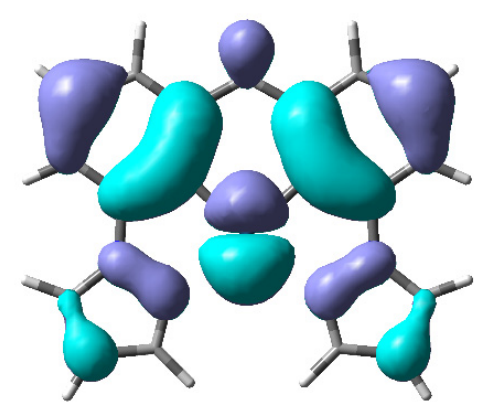

HOMO

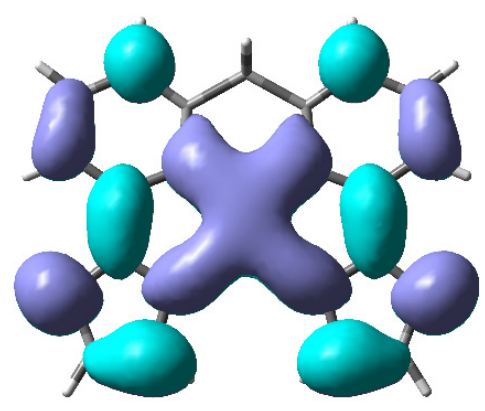

LUMO

Figure S27. Isosurface plots (isovalue $=0.020$ ) of the highest occupied molecular orbital $(\mathrm{HOMO})$ and the lowest unoccupied molecular orbital (LUMO) of PtSN1. 
Table S2. Report of the Cartesian Coordinates of the Optimized Geometry of PtSN1

\begin{tabular}{|c|c|c|c|c|c|}
\hline \multirow{3}{*}{$\begin{array}{c}\text { Center } \\
\text { Number } \\
1\end{array}$} & \multirow{3}{*}{$\begin{array}{l}\text { Atomic } \\
\text { Number } \\
78\end{array}$} & \multirow{2}{*}{$\begin{array}{l}\text { Atomic } \\
\text { Type }\end{array}$} & \multicolumn{3}{|c|}{ Coordinates (Angstroms) } \\
\hline & & & $x$ & Y & $z$ \\
\hline & & 0 & 0.000000 & 0.000000 & 0.000000 \\
\hline 2 & 6 & 0 & 0.000000 & 0.000000 & 1.991217 \\
\hline 3 & 6 & 0 & 1.117340 & 0.000000 & 2.836833 \\
\hline 4 & 6 & 0 & -1.270462 & 0.000216 & 2.606418 \\
\hline 5 & 6 & 0 & 0.931094 & 0.000666 & 4.229262 \\
\hline 6 & 6 & 0 & -1.462582 & 0.000587 & 3.981611 \\
\hline 7 & 6 & 0 & -0.335534 & 0.000941 & 4.800473 \\
\hline 8 & 1 & 0 & 1.801957 & 0.000777 & 4.878626 \\
\hline 9 & 1 & 0 & -2.454022 & 0.000660 & 4.418761 \\
\hline 10 & 1 & 0 & -0.450139 & 0.001364 & 5.878093 \\
\hline 11 & 6 & 0 & 1.984600 & 0.000276 & -0.162134 \\
\hline 12 & 6 & 0 & 2.918401 & -0.000170 & 0.882625 \\
\hline 13 & 6 & 0 & 2.494282 & 0.000968 & -1.478473 \\
\hline 14 & 6 & 0 & 4.291036 & 0.000024 & 0.583594 \\
\hline 15 & 6 & 0 & 3.849260 & 0.001385 & -1.781963 \\
\hline 16 & 6 & 0 & 4.757193 & 0.000822 & -0.725349 \\
\hline 17 & 1 & 0 & 5.009172 & -0.000325 & 1.398677 \\
\hline 18 & 1 & 0 & 4.204207 & 0.002102 & -2.805713 \\
\hline 19 & 1 & 0 & 5.821900 & 0.001075 & -0.927333 \\
\hline 20 & 7 & 0 & -2.111702 & 0.000025 & 0.381507 \\
\hline 21 & 6 & 0 & -3.288658 & -0.000048 & -0.245257 \\
\hline 22 & 6 & 0 & -4.338500 & 0.000042 & 0.686521 \\
\hline 23 & 1 & 0 & -3.330874 & 0.000033 & -1.321902 \\
\hline 24 & 6 & 0 & -3.719953 & -0.000195 & 1.923668 \\
\hline 25 & 1 & 0 & -5.397135 & 0.000058 & 0.488804 \\
\hline 26 & 1 & 0 & -4.133194 & -0.000442 & 2.917355 \\
\hline 27 & 7 & 0 & 0.208225 & 0.000357 & -2.135569 \\
\hline 28 & 6 & 0 & -0.512388 & 0.000633 & -3.257522 \\
\hline 29 & 6 & 0 & 1.614167 & 0.002037 & -3.864175 \\
\hline 30 & 6 & 0 & 0.330716 & 0.001321 & -4.379821 \\
\hline 31 & 1 & 0 & -1.588891 & -0.000004 & -3.211837 \\
\hline 32 & 1 & 0 & 2.570865 & 0.002922 & -4.357037 \\
\hline 33 & 1 & 0 & 0.047367 & 0.001532 & -5.418816 \\
\hline 34 & 7 & 0 & -2.381594 & -0.000148 & 1.710453 \\
\hline 35 & 7 & 0 & 1.510752 & 0.001337 & -2.512892 \\
\hline 36 & 6 & 0 & 2.558016 & -0.001135 & 2.357542 \\
\hline 37 & 1 & 0 & 3.053310 & 0.865985 & 2.813967 \\
\hline 38 & 1 & 0 & 3.051313 & -0.870334 & 2.812232 \\
\hline
\end{tabular}


Table S3. TD-CAM-B3LYP Calculation Results for PtSN2

\begin{tabular}{ccc}
\hline state & energy $(\mathrm{eV})$ & $\begin{array}{c}\text { participating molecular orbitals } \\
\text { (expansion coefficient) }\end{array}$ \\
\hline $\mathrm{T}_{1}$ & 2.93 & $\mathrm{HOMO} \rightarrow$ LUMO $(0.62)$ \\
\hline $\mathrm{T}_{2}$ & 2.96 & $\mathrm{HOMO}-1 \rightarrow$ LUMO $(0.61)$ \\
$\mathrm{S}_{1}$ & 3.24 & $\mathrm{HOMO} \rightarrow$ LUMO $(0.70)$ \\
$\mathrm{T}_{3}$ & 3.33 & $\mathrm{HOMO}-3 \rightarrow$ LUMO $(0.46)$ \\
$\mathrm{T}_{4}$ & 3.44 & $\mathrm{HOMO} \rightarrow$ LUMO $+1(0.57)$ \\
\hline
\end{tabular}

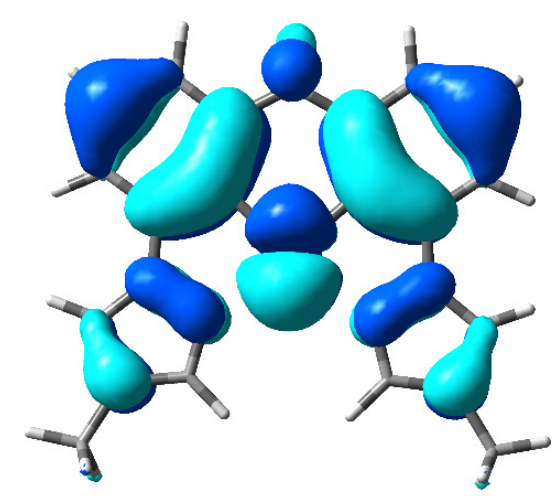

HOMO

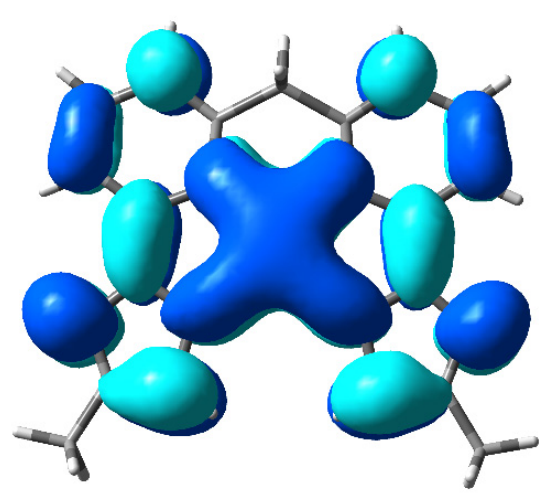

LUMO

Figure S28. Isosurface plots (isovalue $=0.020$ ) of the highest occupied molecular orbital $(\mathrm{HOMO}$ ) and the lowest unoccupied molecular orbital (LUMO) of PtSN2. 
Table S4. Report of the Cartesian Coordinates of the Optimized Geometry of PtSN2

\begin{tabular}{|c|c|c|c|c|c|}
\hline \multirow{2}{*}{$\begin{array}{l}\text { Center } \\
\text { Number }\end{array}$} & \multirow{2}{*}{$\begin{array}{l}\text { Atomic } \\
\text { Number }\end{array}$} & \multirow{2}{*}{$\begin{array}{l}\text { Atomic } \\
\text { Type }\end{array}$} & \multicolumn{3}{|c|}{ Coordinates (Angstroms) } \\
\hline & & & $x$ & $Y$ & Z \\
\hline 1 & 78 & 0 & 0.000000 & 0.000000 & 0.000000 \\
\hline 2 & 6 & 0 & 0.000000 & 0.000000 & 1.991603 \\
\hline 3 & 6 & 0 & 1.121416 & 0.000000 & 2.833496 \\
\hline 4 & 6 & 0 & -1.267292 & 0.042001 & 2.607181 \\
\hline 5 & 6 & 0 & 0.944004 & 0.086715 & 4.222793 \\
\hline 6 & 6 & 0 & -1.452532 & 0.120608 & 3.982498 \\
\hline 7 & 6 & 0 & -0.322225 & 0.151730 & 4.795478 \\
\hline 8 & 1 & 0 & 1.816840 & 0.097085 & 4.868933 \\
\hline 9 & 1 & 0 & -2.442100 & 0.162349 & 4.422145 \\
\hline 10 & 1 & 0 & -0.432602 & 0.223032 & 5.871259 \\
\hline 11 & 6 & 0 & 1.978492 & 0.167540 & -0.154960 \\
\hline 12 & 6 & 0 & 2.909451 & 0.151333 & 0.893558 \\
\hline 13 & 6 & 0 & 2.479848 & 0.359312 & -1.458263 \\
\hline 14 & 6 & 0 & 4.267933 & 0.367960 & 0.616517 \\
\hline 15 & 6 & 0 & 3.824404 & 0.567301 & -1.742681 \\
\hline 16 & 6 & 0 & 4.724990 & 0.578857 & -0.680458 \\
\hline 17 & 1 & 0 & 4.982657 & 0.364853 & 1.434202 \\
\hline 18 & 1 & 0 & 4.174444 & 0.722442 & -2.756426 \\
\hline 19 & 1 & 0 & 5.778865 & 0.748615 & -0.867818 \\
\hline 20 & 7 & 0 & -2.111096 & -0.073641 & 0.388637 \\
\hline 21 & 6 & 0 & -3.289957 & -0.125055 & -0.232685 \\
\hline 22 & 6 & 0 & -4.349358 & -0.091838 & 0.695711 \\
\hline 23 & 6 & 0 & -3.718712 & -0.016164 & 1.926608 \\
\hline 24 & 7 & 0 & 0.213686 & 0.123298 & -2.133655 \\
\hline 25 & 6 & 0 & -0.499015 & 0.111494 & -3.260736 \\
\hline 26 & 6 & 0 & 1.601438 & 0.434635 & -3.845465 \\
\hline 27 & 6 & 0 & 0.331304 & 0.304892 & -4.382572 \\
\hline 28 & 7 & 0 & -2.378019 & -0.005351 & 1.713939 \\
\hline 29 & 7 & 0 & 1.502443 & 0.323369 & -2.496162 \\
\hline 30 & 6 & 0 & 2.540327 & -0.182642 & 2.327176 \\
\hline 31 & 1 & 0 & 3.218516 & 0.358845 & 2.994493 \\
\hline 32 & 1 & 0 & 2.789186 & -1.245111 & 2.473665 \\
\hline 33 & 6 & 0 & -5.822281 & -0.131929 & 0.419653 \\
\hline 34 & 1 & 0 & -6.393490 & -0.113040 & 1.349948 \\
\hline 35 & 1 & 0 & -6.142268 & 0.724107 & -0.181696 \\
\hline
\end{tabular}




\begin{tabular}{llllll}
36 & 1 & 0 & -6.104198 & -1.039029 & -0.122755 \\
37 & 6 & 0 & -0.064075 & 0.356119 & -5.827690 \\
38 & 1 & 0 & 0.810393 & 0.497903 & -6.465881 \\
39 & 1 & 0 & -0.555962 & -0.568957 & -6.142009 \\
40 & 1 & 0 & -0.755183 & 1.180490 & -6.026666 \\
41 & 1 & 0 & -4.132170 & 0.028514 & 2.919980 \\
42 & 1 & 0 & -3.332388 & -0.183761 & -1.308736 \\
43 & 1 & 0 & -1.567018 & -0.033982 & -3.224119 \\
44 & 1 & 0 & 2.549914 & 0.594721 & -4.329713 \\
\hline
\end{tabular}


Table S5. TD-CAM-B3LYP Calculation Results for PtSN3

\begin{tabular}{ccc}
\hline state & energy $(\mathrm{eV})$ & $\begin{array}{c}\text { participating molecular orbitals } \\
\text { (expansion coefficient) }\end{array}$ \\
\hline $\mathrm{T}_{1}$ & 2.98 & $\begin{array}{c}\text { HOMO }-1 \rightarrow \text { LUMO }(0.42) \\
\text { HOMO } \rightarrow \text { LUMO }(0.43)\end{array}$ \\
\hline $\mathrm{T}_{2}$ & 3.04 & $\begin{array}{c}\mathrm{HOMO}-1 \rightarrow \text { LUMO }(0.39) \\
\mathrm{HOMO} \rightarrow \text { LUMO }(0.39)\end{array}$ \\
$\mathrm{T}_{3}$ & 3.38 & $\mathrm{HOMO}-3 \rightarrow$ LUMO $(0.39)$ \\
$\mathrm{S}_{1}$ & 3.39 & $\mathrm{HOMO} \rightarrow$ LUMO $(0.63)$ \\
\hline $\mathrm{T}_{4}$ & 3.44 & HOMO-2 $\rightarrow$ LUMO $(0.59)$ \\
\hline
\end{tabular}

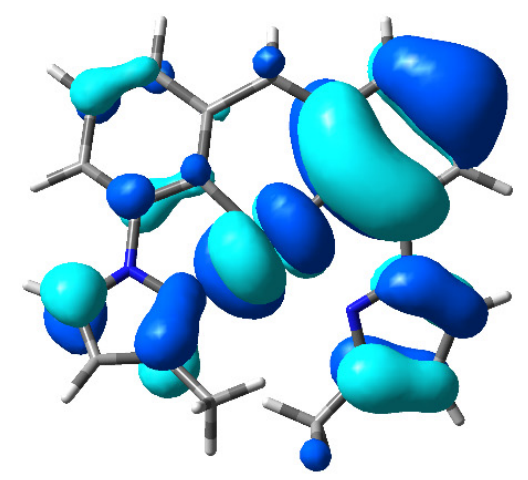

HOMO-1

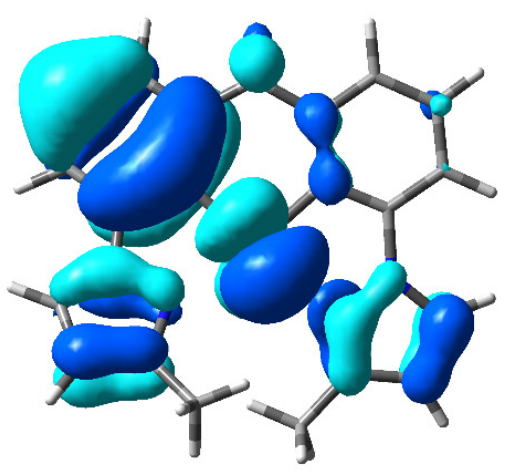

HOMO

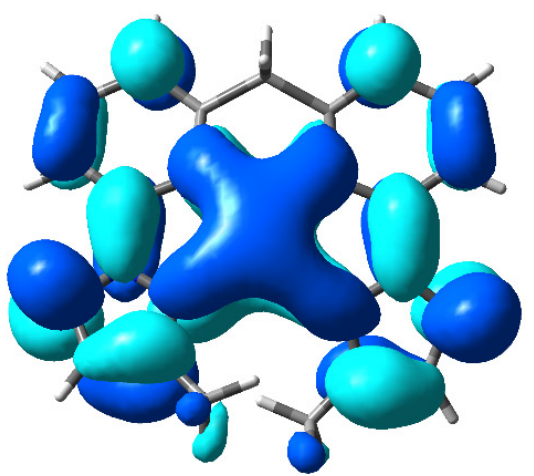

LUMO

Figure S29. Isosurface plots (isovalue $=0.020$ ) of the highest occupied molecular orbital $(\mathrm{HOMO})$, HOMO-1, and the lowest unoccupied molecular orbital (LUMO) of PtSN3. 
Table S6. Report of the Cartesian Coordinates of the Optimized Geometry of PtSN3

\begin{tabular}{|c|c|c|c|c|c|}
\hline \multirow{2}{*}{$\begin{array}{l}\text { Center } \\
\text { Number }\end{array}$} & \multirow{2}{*}{$\begin{array}{l}\text { Atomic } \\
\text { Number }\end{array}$} & \multirow{2}{*}{$\begin{array}{l}\text { Atomic } \\
\text { Type }\end{array}$} & \multicolumn{3}{|c|}{ Coordinates (Angstroms) } \\
\hline & & & $\mathrm{x}$ & $\mathrm{Y}$ & z \\
\hline 1 & 78 & 0 & 0.000000 & 0.000000 & 0.000000 \\
\hline 2 & 6 & 0 & 0.000000 & 0.000000 & 1.990305 \\
\hline 3 & 6 & 0 & 1.120422 & 0.000000 & 2.833935 \\
\hline 4 & 6 & 0 & -1.259184 & 0.173101 & 2.595717 \\
\hline 5 & 6 & 0 & 0.958878 & 0.255638 & 4.202920 \\
\hline 6 & 6 & 0 & -1.429410 & 0.435068 & 3.951005 \\
\hline 7 & 6 & 0 & -0.296197 & 0.489606 & 4.757544 \\
\hline 8 & 1 & 0 & 1.833071 & 0.268788 & 4.846810 \\
\hline 9 & 1 & 0 & -2.411982 & 0.596272 & 4.378744 \\
\hline 10 & 1 & 0 & -0.395872 & 0.702108 & 5.815586 \\
\hline 11 & 6 & 0 & 1.989876 & 0.074437 & -0.135989 \\
\hline 12 & 6 & 0 & 2.910441 & -0.092083 & 0.910711 \\
\hline 13 & 6 & 0 & 2.520516 & 0.239041 & -1.429955 \\
\hline 14 & 6 & 0 & 4.286328 & -0.075630 & 0.638819 \\
\hline 15 & 6 & 0 & 3.882697 & 0.231264 & -1.711796 \\
\hline 16 & 6 & 0 & 4.773764 & 0.077547 & -0.654451 \\
\hline 17 & 1 & 0 & 4.988745 & -0.196492 & 1.458135 \\
\hline 18 & 1 & 0 & 4.253599 & 0.346948 & -2.723177 \\
\hline 19 & 1 & 0 & 5.841276 & 0.079210 & -0.841472 \\
\hline 20 & 7 & 0 & -2.121380 & -0.282813 & 0.423254 \\
\hline 21 & 6 & 0 & -3.264742 & -0.730975 & -0.109016 \\
\hline 22 & 6 & 0 & -4.286214 & -0.687789 & 0.864556 \\
\hline 23 & 6 & 0 & -3.684271 & -0.230193 & 2.016924 \\
\hline 24 & 1 & 0 & -5.316588 & -0.978510 & 0.741224 \\
\hline 25 & 7 & 0 & 0.247526 & 0.467408 & -2.122260 \\
\hline 26 & 6 & 0 & -0.419503 & 0.949984 & -3.179293 \\
\hline 27 & 6 & 0 & 1.732291 & 0.901017 & -3.733892 \\
\hline 28 & 6 & 0 & 0.485239 & 1.216173 & -4.228143 \\
\hline 29 & 1 & 0 & 0.252554 & 1.606962 & -5.205105 \\
\hline 30 & 7 & 0 & -2.381014 & 0.004386 & 1.730558 \\
\hline 31 & 7 & 0 & 1.566551 & 0.457519 & -2.465158 \\
\hline 32 & 6 & 0 & 2.501835 & -0.376010 & 2.343421 \\
\hline 33 & 1 & 0 & 3.245705 & 0.068085 & 3.012142 \\
\hline 34 & 1 & 0 & 2.600647 & -1.462882 & 2.489267 \\
\hline 35 & 1 & 0 & -4.077779 & -0.084880 & 3.008335 \\
\hline
\end{tabular}




\begin{tabular}{llllll}
36 & 1 & 0 & 2.708686 & 0.984825 & -4.178869 \\
37 & 6 & 0 & -3.349837 & -1.281447 & -1.495169 \\
38 & 1 & 0 & -4.009283 & -0.685151 & -2.131062 \\
39 & 1 & 0 & -2.363177 & -1.329098 & -1.953402 \\
40 & 1 & 0 & -3.756685 & -2.295876 & -1.461439 \\
41 & 6 & 0 & -1.888539 & 1.216695 & -3.164876 \\
42 & 1 & 0 & -2.444946 & 0.458962 & -3.723102 \\
43 & 1 & 0 & -2.265967 & 1.247510 & -2.143783 \\
44 & 1 & 0 & -2.090227 & 2.182772 & -3.633970 \\
\hline
\end{tabular}


Table S7. Summary of the Photophysical Properties of the mCBP:TSPO1 Films Doped with the PtSN Compounds

\begin{tabular}{ccccccccccc}
\hline & \multicolumn{3}{c}{ PtSN1 } & \multicolumn{3}{c}{ PtSN2 } & \multicolumn{3}{c}{ PtSN3 } \\
\cline { 2 - 10 } doping \% & 2 wt \% & 4 wt \% & 8 wt $\%$ & 2 wt $\%$ & 4 wt $\%$ & 8 wt \% & 2 wt \% & 4 wt \% & 8 wt \% \\
\hline$\lambda_{\max }{ }^{a}(\mathrm{~nm})$ & 465 & 465 & 578 & 470 & 470 & 567 & 470 & 471 & 472 \\
$h: \nu^{b}$ & $71: 29$ & $71: 29$ & $71: 29$ & $69: 31$ & $69: 31$ & $70: 30$ & $63: 37$ & $64: 36$ & $66: 34$ \\
PLQY $^{c}$ & 0.33 & 0.50 & 0.57 & 0.41 & 0.46 & 0.58 & 0.17 & 0.31 & 0.33 \\
\hline
\end{tabular}

${ }^{a}$ Peak wavelength $\left(\lambda_{\mathrm{ex}}=325 \mathrm{~nm}\right.$ ). ${ }^{b} \mathrm{H}$ orizontal $(h)$ to vertical $(v)$ transition dipole moment ratio from the analysis of angle-dependent photoluminescence measurements. ${ }^{c}$ Absolute photoluminescence quantum yields. 